\title{
Trace and Density Results on Regular Trees
}

\author{
Pekka Koskela $^{1} \cdot$ Khanh Ngoc Nguyen $^{1}$ (D) Zhuang Wang $^{2}$
}

Received: 25 February 2020 / Accepted: 28 January 2021 / Published online: 6 March 2021

(C) The Author(s) 2021

\begin{abstract}
The boundary of a regular tree can be viewed as a Cantor-type set. We equip our tree with a weighted distance and a weighted measure via the Euclidean arc-length and consider the associated first-order Sobolev spaces. We give characterizations for the existence of traces and for the density of compactly supported functions.
\end{abstract}

Keywords Regular tree $\cdot$ Boundary trace $\cdot$ Newtonian space $\cdot$ Density

Mathematics Subject Classification (2010) 46E35 · 30L99

\section{Introduction}

Let $G$ be a $K$-regular tree with a set of vertices $V$ and a set of edges $E$ for some $K \geq 1$. The union of $V$ and $E$ will be denoted by $X$. We abuse the notation and call $X$ a $K$-regular tree. We introduce a metric structure on $X$ by considering each edge of $X$ to be an isometric copy of the unit interval. Then the distance between two vertices is the number of edges needed to connect them and there is a unique geodesic that minimizes this number. Let us denote the root by 0 . If $x$ is a vertex, we define $|x|$ to be the distance between 0 and $x$. Since each edge is an isometric copy of the unit interval, we may extend this distance naturally to any $x$ belonging to an edge. We define $\partial X$ as the collection of all infinite geodesics starting at

All authors have been supported by the Academy of Finland via Centre of Excellence in Analysis and Dynamics Research (project No. 307333). This work was also partially supported by the grant 346300 for IMPAN from the Simons Foundation and the matching 2015-2019 Polish MNiSW fund.

Pekka Koskela

pekka.j.koskela@jyu.fi

Khanh Ngoc Nguyen

khanh.n.nguyen@jyu.fi

Zhuang Wang

zhuang.z.wang@foxmail.com; wangzhuangzhao@gmail.com

1 Department of Mathematics and Statistics, University of Jyväskylä, PO Box 35, FI-40014 Jyväskylä, Finland

2 LCSM(MOE), School of Mathematics and Statistics, Hunan Normal University, 410081, Changsha, China 
the root 0 . Then every $\xi \in \partial X$ corresponds to an infinite geodesic $[0, \xi$ ) (in $X$ ) that is an isometric copy of the interval $[0, \infty)$. Hence $x \rightarrow \xi$ along $[0, \xi)$ has a canonical meaning.

Given a function $f$ defined on $X$, we are interested in the collection of those $\xi \in \partial X$ for which the limit of $f(x)$ exists when $x \rightarrow \xi$ along $[0, \xi)$. We begin by equipping $\partial X$ with the natural probability measure $v$ so that $v\left(I_{x}\right)=K^{-j}$ when $x$ is a vertex with $|x|=j$ and $I_{x}=\{\xi \in \partial X: x \in[0, \xi)\}$. Towards defining the classes of functions that are of interest to us, we define a measure function and a new distance function on $X$. Write $d|x|$ for the length element on $X$ and let $\mu:[0, \infty) \rightarrow(0, \infty)$ be a Borel measurable and locally integrable function. We abuse notation and refer also to the measure generated via $d \mu(x)=\mu(|x|) d|x|$ by $\mu$. Further, let $\lambda:[0, \infty) \rightarrow(0, \infty)$ be Borel measurable and locally integrable, and we define a distance $d$ via $d s(x)=\lambda(|x|) d|x|$ by setting $d(z, y)=\int_{[z, y]} d s(x)$ whenever $z, y \in X$ and $[z, y]$ is the unique geodesic between $z$ and $y$. For convenience, we assume additionally that $\lambda^{p} / \mu \in L_{\mathrm{loc}}^{1 /(p-1)}([0, \infty))$ if $p>1$ below and that $\lambda / \mu \in L_{\mathrm{loc}}^{\infty}([0, \infty))$ if $p=1$. Then $(X, d, \mu)$ is a metric measure space and we let $N^{1, p}(X):=N^{1, p}(X, d, \mu)$, $1 \leq p<\infty$, be the associated Sobolev space based on upper gradients [10], as introduced in [27]. See Section 2 for the precise definition. We show there that, actually, each $u \in$ $N^{1, p}(X)$ is absolutely continuous on each edge, with $u^{\prime} \in L_{\mu}^{p}(X)$. As usual, $N_{0}^{1, p}(X)$ is the completion of the family of functions with compact support in $N^{1, p}(X)$.

In order to state our results, we need two more concepts. Given $1<p<\infty$ we set

$$
R_{p}=\int_{0}^{\infty} \lambda(t)^{\frac{p}{p-1}} \mu(t)^{\frac{1}{1-p}} K^{\frac{t}{1-p}} d t=\left\|\frac{\lambda(x)}{\mu(x) K^{|x|}}\right\|_{L_{\mu}^{\frac{p}{p-1}}(X)}^{\frac{p}{p-1}}
$$

and we define

$$
R_{1}=\left\|\frac{\lambda(t)}{\mu(t) K^{t}}\right\|_{L^{\infty}([0, \infty))} .
$$

One should view $R_{p}$ as an isoperimetric profile $(X, d, \mu)$ : in case of a Riemannian manifold $M$, the natural version of $R_{p}$ is closely related to the parabolicity of the manifold [28]; $R_{p}=\infty$ guarantees parabolicity (every compact set is of relative $p$-capacity zero). This suggests that the existence of limits for Sobolev functions along geodesics might be somehow related to finiteness of $R_{p}$, see Remark 3.8 for a discussion. Let us say that the trace of a given function $f$, defined on $X$, exists if

$$
\operatorname{Tr} f(\xi):=\lim _{[0, \xi) \ni x \rightarrow \xi} f(x)
$$

exists for $v$-a.e. $\xi \in \partial X$. We then denote by $\operatorname{Tr} f$ the trace function of $f$. For other possible definitions of the trace and connections between them see [17].

Our first result gives a rather complete solution for the existence of traces in the case $\mu(X)<\infty$.

Theorem 1.1 Let $X$ be a $K$-regular tree with distance $d$ and measure $\mu$. Assume $\mu(X)<$ $\infty$. For $1 \leq p<\infty$, the following are equivalent:

(i) $R_{p}<\infty$.

(ii) $\operatorname{Tr} f$ exists for every $f \in N^{1, p}(X)$ and $\operatorname{Tr}: N^{1, p}(X) \rightarrow L_{\nu}^{p}(\partial X)$ is a bounded linear operator.

(iii) $\operatorname{Tr} f$ exists for every $f \in N^{1, p}(X)$.

(iv) $N_{0}^{1, p}(X) \subsetneq N^{1, p}(X)$. 
In $[3,18]$ the trace spaces of our Sobolev spaces were identified as suitable Besov-type spaces for very specific choices of $\mu, \lambda$.

For the case $\mu(X)=\infty$, we define

$$
\mathcal{R}_{p}=\sup _{k \geq 1} \int_{A_{k}} \lambda(t)^{\frac{p}{p-1}} \mu(t)^{\frac{1}{1-p}} K^{\frac{t}{1-p}} d t \text { if } p>1 \text { and } \mathcal{R}_{1}=R_{1}
$$

where $\left\{A_{k}\right\}_{k=1}^{\infty}=\left\{\left[t_{k}, t_{k+1}\right)\right\}_{k=1}^{\infty}$ is the sequence of subintervals in $[0, \infty)$ with $\int_{A_{k}} K^{t} \mu(t) d t=1$ for $k=1,2, \ldots$ and $0=t_{1}<t_{2}<\ldots$ Then $[0, \infty)=\bigcup_{k=1}^{\infty} A_{k}$.

Our second result deals with the case of infinite volume.

Theorem 1.2 Let $1 \leq p<\infty$. Let $X$ be a $K$-regular tree with distance $d$ and measure $\mu$. Assume $\mu(X)=\infty$. Then the following hold:

(1) $N_{0}^{1, p}(X)=N^{1, p}(X)$.

(2) The following are equivalent:

(a) $\mathcal{R}_{p}<\infty$.

(b) $\operatorname{Tr} f$ exists and $\operatorname{Tr} f \equiv 0$ for every $f \in N^{1, p}(X)$.

(c) $\operatorname{Tr} f$ exists for every $f \in N^{1, p}(X)$.

In this case of infinite volume and $1<p<\infty$, it is easy to see that $R_{p}<\infty$ implies $\mathcal{R}_{p}<\infty$, but the inverse implication need not hold true. Moreover, the finiteness of $\mathcal{R}_{p}$ does not imply the finiteness of $R_{q}$ for some $1 \leq q<\infty$, see Example 3.14, and Remark 3.15 .

Our third result gives a complete answer in the case of homogeneous norms, see Section 2 for the relevant definitions. Here $\dot{N}_{0}^{1, p}(X)$ is the completion of the family of functions with compact support in $\dot{N}^{1, p}(X)$.

Theorem 1.3 Let $X$ be a $K$-regular tree with distance $d$ and measure $\mu$. For $1 \leq p<\infty$, the following are equivalent:

(i) $R_{p}<\infty$.

(ii) $\operatorname{Tr} f$ exists for every $f \in \dot{N}^{1, p}(X)$ and $\operatorname{Tr}: \dot{N}^{1, p}(X) \rightarrow L_{v}^{p}(\partial X)$ is a bounded linear operator.

(iii) $\operatorname{Tr} f$ exists for every $f \in \dot{N}^{1, p}(X)$.

(iv) $\dot{N}_{0}^{1, p}(X) \subsetneq \dot{N}^{1, p}(X)$.

Let us close this introduction with some comments on Theorem 1.3. Even though the condition $R_{p}=\infty$ implies $p$-parabolicity, finiteness of this quantity does not, in general, prevent $p$-parabolicity, see [7]. Hence Theorem 1.3 and the preceding theorems are somewhat surprising. In fact, it follows from our results that, in the setting of this paper, $R_{p}=\infty$ precisely when $(X, d, \mu)$ is $p$-parabolic. See [23] for more on this. Hence the reader familiar with moduli of curve families might wish to view Theorem 1.3 as kind of a version of the equivalence between modulus and capacity.

Partial motivation for this paper comes from boundary value problems for the $p$-Laplace equation. For the case of manifolds see $[12,13]$ and for the setting of metric spaces see $[2$, $4,15]$. Classical trace results on the Euclidean spaces can be found in $[1,6,9,14,16,22$, $25,29,30]$ and studies of parabolicity on infinite networks in [26, 32]. For trace results in the metric setting see $[3,18-21,33]$. Our second motivation comes from the recent paper [24] where a version of Theorem 1.3 was established on regular trees for the case $p=2$. 
The paper is organized as follows. In Section 2, we introduce regular trees, boundaries of trees and Newtonian spaces on our trees. We study the trace results in Section 3 and the density results are given in Section 4. In Section 5, we give the proofs of Theorems 1.1-1.3.

\section{Preliminaries}

Throughout this paper, the letter $C$ (sometimes with a subscript) will denote positive constants that usually depend only on the space and may change at different occurrences; if $C$ depends on $a, b, \ldots$, we write $C=C(a, b, \ldots)$. The notation $A \lesssim B(A \gtrsim B)$ means that there is a constant $C$ such that $A \leq C \cdot B(A \geq C \cdot B)$. The notation $A \approx B$ means that both $A \lesssim B$ and $B \lesssim A$ hold. For any function $f \in L_{\text {loc }}^{1}(X)$ and any measurable subset $A \subset X$ with $\mu(A)>0$, we denote $\frac{1}{\mu(A)} \int_{A} f d \mu$ by $f_{A} f d \mu$.

\subsection{Regular Trees and Their Boundaries}

A graph $G$ is a pair $(V, E)$, where $V$ is a set of vertices and $E$ is a set of edges. We call a pair of vertices $x, y \in V$ neighbors if $x$ is connected to $y$ by an edge. The degree of a vertex is the number of its neighbors. The graph structure gives rise to a natural connectivity structure. A tree $G$ is a connected graph without cycles.

We call a tree $G$ a rooted tree if it has a distinguished vertex called the root, which we will denote by 0 . The neighbors of a vertex $x \in V$ are of two types: the neighbors that are closer to the root are called parents of $x$ and all other neighbors are called children of $x$. Each vertex has a unique parent, except for the root itself that has none.

We say that a tree $G$ is $K$-regular if $G$ is a rooted tree such that each vertex has exactly $K$ children for some $K \geq 1$. Then all vertices except the root of $G$ have degree $K+1$, and the root has degree $K$.

Let $G$ be a $K$-regular tree with a set of vertices $V$ and a set of edges $E$ for some $K \geq 1$. For simplicity of notation, we let $X=V \cup E$ and call it a $K$-regular tree. A $K$-regular tree $X$ is made into a metric graph by considering each edge as a geodesic of length one. For $x \in X$, let $|x|$ be the distance from the root 0 to $x$, that is, the length of the geodesic from 0 to $x$, where the length of every edge is 1 and we consider each edge to be an isometric copy of the unit interval. The geodesic connecting $x, y \in V$ is denoted by $[x, y]$, and its length is denoted by $|x-y|$. If $|x|<|y|$ and $x$ lies on the geodesic connecting 0 to $y$, we write $x<y$ and call the vertex $y$ a descendant of the vertex $x$. More generally, we write $x \leq y$ if the geodesic from 0 to $y$ passes through $x$, and in this case $|x-y|=|y|-|x|$.

On the $K$-regular tree $X$, for any $n \in \mathbb{N}$, let $X^{n}$ be a subset of $X$ by setting

$$
X^{n}:=\{x \in X:|x|<n\} .
$$

On the $K$-regular tree $X$, we define a metric $d$ via $d s$ and measure $d \mu$ by setting

$$
d \mu=\mu(|x|) d|x|, \quad d s(x)=\lambda(|x|) d|x|,
$$

where $\lambda, \mu:[0, \infty) \rightarrow(0, \infty)$ are Borel functions with $\lambda, \mu \in L_{\text {loc }}^{1}([0, \infty))$. Throughout this paper, we let $1 \leq p<\infty$ and assume additionally that $\lambda^{p} / \mu \in L_{\text {loc }}^{1 /(p-1)}([0, \infty))$ if $p>1$ and that $\lambda / \mu \in L_{\mathrm{loc}}^{\infty}([0, \infty))$ if $p=1$. Here $d|x|$ is the measure which gives each edge Lebesgue measure 1 , as we consider each edge to be an isometric copy of the 
unit interval and the vertices are the end points of this interval. Hence for any two points $z, y \in X$, the distance between them is

$$
d(z, y)=\int_{[z, y]} d s(x)=\int_{[z, y]} \lambda(|x|) d|x|,
$$

where $[z, y]$ is the unique geodesic from $z$ to $y$ in $X$.

We abuse the notation and let $\mu(x)$ and $\lambda(x)$ denote $\mu(|x|)$ and $\lambda(|x|)$, respectively, for any $x \in X$, if there is no danger of confusion.

Next we construct the boundary of the $K$-regular tree. An element $\xi$ in $\partial X$ is identified with an infinite geodesic in $X$ starting at the root 0 . Then we may denote $\xi=0 x_{1} x_{2} \cdots$, where $x_{i}$ is a vertex in $X$ with $\left|x_{i}\right|=i$, and $x_{i+1}$ is a child of $x_{i}$. Given two points $\xi, \zeta \in \partial X$, there is an infinite geodesic $[\xi, \zeta]$ connecting $\xi$ and $\zeta$.

To avoid confusion, points in $X$ are denoted by Latin letters such as $x, y$ and $z$, while for points in $\partial X$ we use Greek letters such as $\xi, \zeta$ and $\omega$.

We equip $\partial X$ with the natural probability measure $v$ as in Falconer [5] by distributing the unit mass uniformly on $\partial X$. For any $x \in X$ with $|x|=j$, if we denote by $I_{x}$ the set

$$
\{\xi \in \partial X: \text { the geodesic }[0, \xi) \text { passes through } x\},
$$

then the measure of $I_{x}$ is $K^{-j}$. We refer to [3, Lemma 5.2] for a more information on our boundary measure $v$.

\subsection{Newtonian Spaces}

Let $X$ be a $K$-regular tree with metric and measure defined as in Section 2.1. Let $\mathscr{M}$ denote the family of all nonconstant rectifiable curves in $X$. We recall the definition of $p$-modulus of curve families in $\mathscr{M}$, see $[8,11]$ for more detailed discussions.

Definition 2.1 For $\Gamma \subset \mathscr{M}$, let $F(\Gamma)$ be the family of all Borel measurable functions $\rho: X \rightarrow[0, \infty]$ such that

$$
\int_{\gamma} \rho d s \geq 1 \text { for every } \gamma \in \Gamma \text {. }
$$

For $1 \leq p<\infty$, we define

$$
\operatorname{Mod}_{p}(\Gamma)=\inf _{\rho \in F(\Gamma)} \int_{X} \rho^{p} d \mu .
$$

The number $\operatorname{Mod}_{p}(\Gamma)$ is called the $p$-modulus of the family $\Gamma$.

Proposition 2.2 Let $1 \leq p<\infty$. Then the following are equivalent:

1. $\lambda / \mu^{1 / p} \in L_{\text {loc }}^{p^{\prime}}([0, \infty))$ where $p^{\prime}$ is the Hölder conjugate of $p$, that is $\frac{1}{p}+\frac{1}{p^{\prime}}=1$.

2. $\operatorname{Mod}_{p}(\{\gamma\})>0$ for every rectifiable curve $\gamma$.

In particular, on our $K$-regular tree $X$ with metric and measure defined as in Section 2.1, the empty family is the only curve family with zero $p$-modulus for $1 \leq p<\infty$. 
Proof 1. $\Rightarrow$ 2.: For every Borel measurable function $\rho \in F(\{\gamma\})$, we have $\int_{\gamma} \rho d s \geq 1$. By the monotone convergence theorem, we may assume that $\int_{\gamma} \rho d s \geq 1 / 2$ for a subcurve, still denoted $\gamma$, that is contained in $\{x \in X:|x| \leq N\}$ for some $N \in \mathbb{N}$. Notice that

$$
d s(x)=\frac{\lambda(x)}{\mu(x)} d \mu(x) .
$$

For $p>1$, it follows from the Hölder inequality that

$$
\begin{gathered}
\int_{\gamma} \rho d s=\int_{\gamma} \rho \frac{\lambda}{\mu} d \mu \leq \\
\left.\leq \int_{\gamma} \rho^{p} d \mu\right)^{1 / p}\left(\int_{\gamma} \frac{\lambda^{p /(p-1)}}{\mu^{p /(p-1)}} d \mu\right)^{(p-1) / p} \\
\leq C(N, p, \lambda, \mu, K)\left(\int_{\gamma} \rho^{p} d \mu\right)^{1 / p},
\end{gathered}
$$

since it follows from $\lambda^{p} / \mu \in L_{\mathrm{loc}}^{1 /(p-1)}([0, \infty))$ that

$$
0<\int_{\gamma} \frac{\lambda^{p /(p-1)}}{\mu^{p /(p-1)}} d \mu \leq K^{N} \int_{0}^{N}\left(\frac{\lambda(t)^{p}}{\mu(t)}\right)^{\frac{1}{p-1}} d t<\infty .
$$

Hence we have that

$$
\int_{X} \rho^{p} d \mu \geq \int_{\gamma} \rho^{p} d \mu \geq C(N, p, \lambda, \mu, K)\left(\int_{\gamma} \rho d s\right)^{p} \geq C(N, p, \lambda, \mu, K) / 2>0 .
$$

For the case $p=1$, by a similar argument without using the Hölder inequality, it follows from $\lambda / \mu \in L_{\mathrm{loc}}^{\infty}([0, \infty))$ that

$$
\int_{X} \rho d \mu \geq C(N, \lambda, \mu, K)>0 .
$$

Thus

$$
\operatorname{Mod}_{p}(\{\gamma\})>0 \text { for every rectifiable curve } \gamma .
$$

2. $\Rightarrow$ 1.: We argue by contradiction. Assume that $\frac{\lambda}{\mu^{1 / p}} \notin L_{\mathrm{loc}}^{p^{\prime}}([0, \infty))$. Then it suffices to find a rectifiable curve $\gamma$ with $\operatorname{Mod}_{p}(\{\gamma\})=0$.

Since $\frac{\lambda}{\mu^{1 / p}} \notin L_{\mathrm{loc}}^{p^{\prime}}([0, \infty))$, there exist two constants $0 \leq a<b<\infty$ such that

$$
\left\|\frac{\lambda}{\mu^{1 / p}}\right\|_{L^{p^{\prime}([a, b])}}=\infty .
$$

Let $x_{a}, y_{b} \in X$ with $\left|x_{a}\right|=a,\left|y_{b}\right|=b$ and $x_{a} \leq y_{b}$. Then we claim that the geodesic $\left[x_{a}, y_{b}\right]$ from $x_{a}$ to $y_{b}$, denoted by $\gamma$, satisfies $\operatorname{Mod}_{p}(\{\gamma\})=0$.

To prove the above claim, by [8, Theorem 5.5] or [11, Lemma 5.2.8], we only need to find a Borel measurable function $0 \leq \rho \in L^{p}(X)$ such that

$$
\int_{\gamma} \rho d s=\infty
$$

For $p>1$, the condition Eq. 2.3 implies

$$
\int_{a}^{b} \frac{\lambda^{\frac{p}{p-1}}(t)}{\mu^{\frac{1}{p-1}}(t)} d t=\infty
$$


Since $\lambda, \mu$ are Borel measurable functions, there is a sequence of pairwise disjoint Borel subsets $\left\{I_{k}: I_{k} \subset[a, b]\right\}_{k=1}^{\infty}$ such that

$$
2^{k}<\int_{I_{k}} \lambda^{\frac{p}{p-1}}(t) \mu^{\frac{1}{1-p}}(t) d t<\infty
$$

We define a function $\rho: X \rightarrow \mathbb{R}$ by setting

$$
\rho(x)=h(|x|) \chi_{\gamma}(x)=h(|x|) \chi_{\left[x_{a}, y_{b}\right]}(x)
$$

where

$$
h(t):=\sum_{k=1}^{\infty} \frac{\lambda^{\frac{1}{p-1}}(t) \mu^{\frac{1}{1-p}}(t)}{\int_{I_{k}} \lambda^{\frac{p}{p-1}}(t) \mu^{\frac{1}{1-p}}(t) d t} \chi_{I_{k}(t)} .
$$

Since $\lambda, \mu$ are Borel measurable functions and $I_{k}$ is Borel for each $k$, the function $\rho$ is Borel measurable. Moreover, since the subsets $\left\{I_{k}\right\}_{k=1}^{\infty}$ are pairwise disjoint, we obtain the $L^{p}$-estimate

$$
\begin{aligned}
\|\rho\|_{L^{p}(X)}^{p} & =\int_{\left[x_{a}, y_{b}\right]}|h(|x|)|^{p} d \mu(x)=\int_{a}^{b} h(t)^{p} \mu(t) d t \\
& =\sum_{k=1}^{\infty} \int_{I_{k}}\left(\frac{\lambda^{\frac{1}{p-1}}(t) \mu^{\frac{1}{1-p}}(t)}{\int_{I_{k}} \lambda^{\frac{p}{p-1}}(t) \mu^{\frac{1}{1-p}}(t) d t}\right)^{p} \mu(t) d t \\
& =\sum_{k=1}^{\infty} \frac{1}{\left(\int_{I_{k}} \lambda^{\frac{p}{p-1}}(t) \mu^{\frac{1}{1-p}}(t) d t\right)^{p-1}}<\sum_{k=1}^{\infty} \frac{1}{2^{k(p-1)}}<\infty .
\end{aligned}
$$

Finally, Eq. 2.4 follows from

$$
\int_{\gamma} \rho d s=\int_{a}^{b} h(t) \lambda(t) d t=\sum_{k=1}^{\infty} \int_{I_{k}} \frac{\lambda^{\frac{1}{p-1}}(t) \mu^{\frac{1}{1-p}}(t)}{\int_{I_{k}} \lambda^{\frac{p}{p-1}}(t) \mu^{\frac{1}{1-p}}(t) d t} \lambda(t) d t=\sum_{k=1}^{\infty} 1=\infty .
$$

For $p=1$, the condition Eq. 2.3 implies

$$
\left\|\frac{\lambda}{\mu^{1 / p}}\right\|_{L^{\infty}([a, b])}=\infty .
$$

Using a similar argument as for Theorem 3.5 for the case $p=1$ below, there exists an infinite sequence $\left\{E_{k_{n}}\right\}_{k_{n} \in \mathbb{N}}$ defined by

$$
E_{k_{n}}=\left\{t \in[a, b]: 2^{k_{n+1}} \geq \frac{\lambda(t)}{\mu(t)}>2^{k_{n}}\right\}
$$

such that

$$
\left|E_{k_{n}}\right|>0 \text { for each } k_{n} \in \mathbb{N} .
$$

We define a function $\rho: X \rightarrow \mathbb{R}$ by setting

$$
\rho(x)=\tilde{h}(|x|) \chi_{\gamma}(x)=\tilde{h}(|x|) \chi_{\left[x_{a}, y_{b}\right]}(x),
$$

where

$$
\tilde{h}(t)=\sum_{k=1}^{\infty} \frac{1}{\int_{E_{k_{n}}} \lambda(t) d t} \chi_{E_{k_{n}}}(t)
$$


Notice that the subsets $\left\{E_{k_{n}}\right\}_{k_{n} \in \mathbb{N}}$ are Borel and pairwise disjoint. Hence the function $\rho$ is Borel measurable and

$$
\begin{aligned}
\|\rho\|_{L^{1}(X)} & =\int_{\left[x_{a}, y_{b}\right]}|\tilde{h}(|x|)| d \mu(x)=\int_{a}^{b} \tilde{h}(t) \mu(t) d t \\
& =\sum_{k_{n} \in \mathbb{N}} \int_{E_{k_{n}}} \frac{\mu(t)}{\int_{E_{k_{n}}} \lambda(t) d t} d t \\
& <\sum_{k_{n} \in \mathbb{N}} \frac{\int_{E_{k_{n}}} 2^{-k_{n}} \lambda(t) d t}{\int_{E_{k_{n}}} \lambda(t) d t}=\sum_{k_{n} \in \mathbb{N}} 2^{-k_{n}}<\infty .
\end{aligned}
$$

Moreover, Eq. 2.4 holds since

$$
\int_{\gamma} \rho d s=\int_{a}^{b} \tilde{h}(t) \lambda(t) d t=\sum_{k_{n} \in \mathbb{N}} \int_{E_{k_{n}}} \frac{1}{\int_{E_{k_{n}}} \lambda(t) d t} d s=\sum_{k_{n} \in \mathbb{N}} 1=\infty .
$$

Finally, the last claim follows since $\lambda / \mu^{\frac{1}{p}} \in L_{\text {loc }}^{p^{\prime}}([0, \infty))$.

Let $u \in L_{\text {loc }}^{1}(X)$. We say that a Borel function $g: X \rightarrow[0, \infty]$ is an upper gradient of $u$ if

$$
|u(z)-u(y)| \leq \int_{\gamma} g d s
$$

whenever $z, y \in X$ and $\gamma$ is the geodesic from $z$ to $y$. In the setting of a tree any rectifiable curve with end points $z$ and $y$ contains the geodesic connecting $z$ and $y$, and therefore the upper gradient defined above is equivalent to the definition which requires that inequality Eq. 2.5 holds for all rectifiable curves with end points $z$ and $y$. In $[8,11]$, the notion of $p$ weak upper gradient is given. A Borel function $g: X \rightarrow[0, \infty]$ is called a $p$-weak upper gradient of $u$ if Eq. 2.5 holds on $p$-a.e. curves $\gamma \in \mathscr{M}$, i.e., Eq. 2.5 holds for all curves $\gamma \in \mathscr{M} \backslash \Gamma$, where $\operatorname{Mod}_{p}(\Gamma)=0$. Notice that by Proposition 2.2, any $p$-weak upper gradient is actually an upper gradient here. We refer to $[8,11]$ for more information about $p$-weak upper gradients.

The notion of upper gradients is due to Heinonen and Koskela [10]; we refer interested readers to $[2,8,11,27]$ for a more detailed discussion on upper gradients.

The Newtonian space $N^{1, p}(X), 1 \leq p<\infty$, is defined as the collection of all functions $u$ for which the $N^{1, p}$-norm of $u$ defined as

$$
\|u\|_{N^{1, p}(X)}:=\|u\|_{L^{p}(X)}+\inf _{g}\|g\|_{L^{p}(X)}<\infty,
$$

where the infimum is taken over all upper gradients of $u$. If $u \in N^{1, p}(X)$, then it has a minimal $p$-weak upper gradient, which is an upper gradient in our case (by Proposition 2.2). We denote by $g_{u}$ the minimal upper gradient, which is unique up to measure zero and which is minimal in the sense that if $g \in L^{p}(X)$ is any upper gradient of $u$ then $g_{u} \leq g$ a.e. We refer to [8, Theorem 7.16] for proofs of the existence and uniqueness of such minimal upper gradient. Throughout this paper, we denote by $g_{u}$ the (minimal) upper gradient of $u$.

By Proposition 2.2, it follows from [8, Definition 7.2 and Lemma 7.6] that any function $u \in L_{\text {loc }}^{1}(X)$ with an upper gradient $0 \leq g \in L^{p}(X)$ is locally absolutely continuous, for example, absolutely continuous on each edge. Moreover, the "classical" derivative $u^{\prime}$ of this locally absolutely continuous function is a minimal upper gradient in the sense that $g_{u}=\left|u^{\prime}(x)\right| / \lambda(x)$ when $u$ is parameterized in the nature way. 
We define the homogeneous Newtonian spaces $\dot{N}^{1, p}(X), 1 \leq p<\infty$, the collection of all continuous functions $u$ that have an upper gradient $0 \leq g \in L^{p}(X)$, for which the homogeneous $\dot{N}^{1, p}$-norm of $u$ defined as

$$
\|u\|_{\dot{N}^{1, p}(X)}:=|u(0)|+\inf _{g}\|g\|_{L^{p}(X)}
$$

is finite. Here 0 is the root of the $K$-regular tree $X$ and the infimum is taken over all upper gradients of $u$.

\section{Trace Results}

In this section, if we do not specifically mention, we always assume that $X$ is a $K$-regular tree with measure and metric as in Section 2.1.

Lemma 3.1 Let $1 \leq p<\infty$. For every $f \in L^{p}(X)$, we have that

$$
\int_{\partial X} \int_{[0, \xi)}|f(x)|^{p} K^{j(x)} d \mu(x) d v(\xi) \approx \int_{X}|f(x)|^{p} d \mu(x),
$$

where $j(x)$ is the largest integer such that $j(x) \leq|x|+1$.

Proof Let $f \in L^{p}(X)$. For any $\xi \in \partial X$, let $x_{j}=x_{j}(\xi)$ be the ancestor of $\xi$ with $\left|x_{j}\right|=j$. Then it follows from Fubini's Theorem that

$$
\begin{aligned}
\int_{\partial X} \int_{[0, \xi)}|f(x)|^{p} K^{j(x)} d \mu(x) d \nu(\xi)= & \int_{\partial X} \sum_{j=0}^{+\infty} \int_{\left[x_{j}(\xi), x_{j+1}(\xi)\right]}|f(x)|^{p} K^{j} d \mu(x) d \nu(\xi) \\
= & \int_{X}|f(x)|^{p} \int_{\partial X} \sum_{j=0}^{+\infty} K^{j} \\
& \chi_{\left[x_{j}(\xi), x_{j+1}(\xi)\right]}(x) d \nu(\xi) d \mu(x) .
\end{aligned}
$$

Note that $\chi_{\left[x_{j}(\xi), x_{j+1}(\xi)\right]}(x)$ is nonzero only if $j \leq|x| \leq j+1$ and $x<\xi$. Thus the above equality can be rewritten as

$$
\int_{\partial X} \int_{[0, \xi)}|f(x)|^{p} K^{j(x)} d \mu(x) d \nu(\xi)=\int_{X}|f(x)|^{p} K^{j(x)} v\left(I_{x}\right) d \mu(x)
$$

where $I_{x}=\{\xi \in \partial X: x<\xi\}$. Since $v\left(I_{x}\right) \approx K^{-j(x)}$, we obtain that

$$
\int_{\partial X} \int_{[0, \xi)}|f(x)|^{p} K^{j(x)} d \mu(x) d \nu(\xi) \approx \int_{X}|f(x)|^{p} d \mu(x) .
$$

Theorem 3.2 Let $1 \leq p<\infty$ and assume that $R_{p}<+\infty$. Then the trace $\operatorname{Tr}$ in Eq. 1.1 gives a bounded linear operator $\operatorname{Tr}: \dot{N}^{1, p}(X) \rightarrow L_{v}^{p}(\partial X)$.

Proof Let $f \in \dot{N}^{1, p}(X)$. Our task is to show that

$$
\operatorname{Tr} f(\xi):=\tilde{f}(\xi)=\lim _{[0, \xi) \ni x \rightarrow \xi} f(x),
$$

exists for $v$-a.e. $\xi \in \partial X$ and that the trace $\operatorname{Tr} f$ satisfies the norm estimates. 
To show that the limit in Eq. 3.1 exists for $v$-a.e. $\xi \in \partial X$, it suffices to show that the function

$$
\tilde{f}^{*}(\xi)=|f(0)|+\int_{[0, \xi)} g_{f} d s
$$

is in $L_{v}^{p}(\partial X)$, where $[0, \xi)$ is the geodesic ray from 0 to $\xi$ and $g_{f}$ is the minimal upper gradient of $f$. To be more precise, if $\tilde{f}^{*} \in L_{v}^{p}(\partial X)$, we have $\left|\tilde{f}^{*}\right|<\infty$ for $v$-a.e. $\xi \in \partial X$, and hence the limit in Eq. 3.1 exists for $v$-a.e. $\xi \in \partial X$.

Since we have

$$
d s=\frac{\lambda(x)}{\mu(x)} d \mu
$$

we obtain the estimate

$$
\tilde{f}^{*}(\xi)=|f(0)|+\int_{[0, \xi)} g_{f} d s=|f(0)|+\int_{[0, \xi)} g_{f} \frac{\lambda(x)}{\mu(x)} d \mu .
$$

For $p>1$, it follows from the Hölder inequality that

$$
\begin{gathered}
\left|\tilde{f}^{*}(\xi)\right|^{p} \\
\lesssim|f(0)|^{p}+\left(\int_{[0, \xi)} g_{f} K^{j(x) / p} \frac{\lambda(x)}{\mu(x) K^{j(x) / p}} d \mu\right)^{p} \\
\leq|f(0)|^{p}+\int_{[0, \xi)} g_{f}^{p} K^{j(x)} d \mu\left(\int_{[0, \xi)}\left(\frac{\lambda(x)}{\mu(x) K^{j(x) / p}}\right)^{\frac{p}{p-1}} d \mu\right)^{p-1} \\
\lesssim|f(0)|^{p}+R_{p}^{p-1} \int_{[0, \xi)} g_{f}^{p} K^{j(x)} d \mu,
\end{gathered}
$$

where $j(x)$ is the largest integer such that $j(x) \leq|x|+1$. Here the last inequality holds since

$$
\int_{[0, \xi)}\left(\frac{\lambda(x)}{\mu(x) K^{j(x) / p}}\right)^{\frac{p}{p-1}} d \mu \approx \int_{0}^{\infty} \frac{\lambda(t)^{\frac{p}{p-1}}}{\mu(t)^{\frac{p}{p-1}} K^{\frac{t}{p-1}}} \mu(t) d t=R_{p} .
$$

Integrating over all $\xi \in \partial X$, since $v(\partial X)=1, R_{p}<+\infty$ and $g_{f} \in L^{p}(X)$, it follows from Lemma 3.1 that

$$
\begin{gathered}
\int_{\partial X}\left|\tilde{f}^{*}(\xi)\right|^{p} d \nu \lesssim|f(0)|^{p}+\int_{\partial X} \int_{[0, \xi)} g_{f}(x)^{p} K^{j(x)} d \mu(x) d \nu(\xi) \\
\lesssim|f(0)|^{p}+\int_{X} g_{f}(x)^{p} d \mu(x), \quad p>1 .
\end{gathered}
$$

For $p=1$, integrating over all $\xi \in \partial X$ with respect to estimate Eq. 3.2, since $\nu(\partial X)=1$, we obtain by means of Fubini's theorem that

$$
\begin{aligned}
\int_{\partial X}\left|\tilde{f}^{*}(\xi)\right| d \nu & \leq|f(0)|+\int_{\partial X} \int_{X} g_{f}(x) \chi_{[0, \xi)}(x) \frac{\lambda(x)}{\mu(x)} d \mu(x) d v(\xi) \\
& =|f(0)|+\int_{X} g_{f}(x) \frac{\lambda(x)}{\mu(x)} \int_{\partial X} \chi_{[0, \xi)}(x) d \nu(\xi) d \mu(x) \\
& =|f(0)|+\int_{X} g_{f}(x) \lambda(x) \mu(x)^{-1} v\left(I_{x}\right) d \mu(x) .
\end{aligned}
$$

Here in the above estimates, the notations $I_{x}$ and $j(x)$ are the same ones as those we used in Lemma 3.1. Since $v\left(I_{x}\right) \approx K^{-|x|}$ and $R_{1}<+\infty$, we further obtain that

$$
\int_{\partial X}\left|\tilde{f}^{*}(\xi)\right| d \nu \lesssim|f(0)|+R_{1} \int_{X} g_{f}(x) d \mu(x) \lesssim|f(0)|+\int_{X} g_{f}(x) d \mu(x) .
$$


Hence we obtain from estimates Eq. 3.3 and Eq. 3.4 that $\tilde{f}^{*}$ is in $L_{v}^{p}(\partial X)$ for $1 \leq p<$ $\infty$, which gives the existence of the limits in Eq. 3.1 for $v$-a.e. $\xi \in \partial X$. In particular, since $|\tilde{f}| \leq \tilde{f}^{*}$, we have the estimate

$$
\int_{\partial X}|\tilde{f}|^{p} d \nu \lesssim|f(0)|^{p}+\int_{X} g_{f}^{p} d \mu
$$

and hence the norm estimate

$$
\|\tilde{f}\|_{L_{\nu}^{p}(\partial X)} \lesssim|f(0)|+\left(\int_{X} g_{f}^{p} d \mu\right)^{1 / p}=\|f\|_{\dot{N}^{1, p}(X)} .
$$

Since every $f \in N^{1, p}(X)$ is locally absolutely continuous, a direct computation gives the estimate $|f(0)| \lesssim\|f\|_{N^{1, p}(X)}$. Hence we obtain the following result from the above theorem.

Corollary 3.3 Let $1 \leq p<\infty$ and assume that $R_{p}<+\infty$. Then the trace $\operatorname{Tr}$ in Eq. 1.1 gives a bounded linear operator $\operatorname{Tr}: N^{1, p}(X) \rightarrow L_{v}^{p}(\partial X)$.

Next, we study non-existence of the traces when $R_{p}=\infty$. Before going to the main theorems, we introduce the following lemma.

Lemma 3.4 ([31]) Let $\left(\Omega, d, \mu_{\Omega}\right)$ be a $\sigma$-finite metric measure space. Then the following conditions on $\left(\Omega, d, \mu_{\Omega}\right)$ are equivalent:

(i) $L^{p}(\Omega) \subset L^{q}(\Omega)$ for all $p, q \in(0, \infty)$ with $p>q$;

(ii) $\mu(\Omega)<+\infty$.

Theorem 3.5 Let $1 \leq p<\infty$ and assume that $R_{p}=+\infty$. Then there exists a function $u \in \dot{N}^{1, p}(X)$ such that

$$
\lim _{[0, \xi) \ni x \rightarrow \xi} u(x)=+\infty, \text { for all } \xi \in \partial X .
$$

Proof To construct the function $u \in \dot{N}^{1, p}(X)$ satisfying Eq. 3.5, it suffices to find a nonnegative measurable function $g:[0, \infty) \rightarrow[0, \infty]$ such that

$$
\left\{\begin{array}{l}
\int_{0}^{+\infty} g(t) \lambda(t) d t=+\infty \\
\int_{0}^{+\infty} g(t)^{p} \mu(t) K^{t} d t<+\infty
\end{array}\right.
$$

Given such $g$, we may define the function $u$ by setting $u(0)=0$ and $u(x)=\int_{0}^{|x|} g(t) \lambda(t) d t$ for any $x \in X$. Then it follows from the definition of upper gradient that $g_{u}: X \rightarrow[0, \infty]$ defined by $g_{u}(x)=g(|x|)$ is an upper gradient of $u$. Moreover, we obtain that

$$
\left\|g_{u}\right\|_{L^{p}(X)}^{p}=\int_{X} g_{u}^{p} d \mu \approx \int_{0}^{+\infty} g(t)^{p} \mu(t) K^{t} d t<+\infty .
$$

Hence the condition Eq. 3.6 implies $u \in \dot{N}^{1, p}(X)$ and that Eq. 3.5 holds.

$$
\begin{aligned}
& \text { For } p=1 \text {, since } R_{1}=\left\|\frac{\lambda(t)}{\mu(t) K^{t}}\right\|_{L^{\infty}([0, \infty))}=\infty \text {, the sets } \\
& \qquad A_{k}:=\left\{t \in[0, \infty): \frac{\lambda(t)}{\mu(t) K^{t}} \geq 2^{k}\right\}, \quad k \in \mathbb{N}
\end{aligned}
$$


form a nonincreasing sequence of subsets of $[0, \infty)$ and we have

$$
\left|A_{k}\right|>0 \text { for any } k \in \mathbb{N} \text {. }
$$

Hence there exists an infinite sequence $\left\{k_{n}\right\}_{k_{n} \in \mathbb{N}}$ such that $\left|B_{k_{n}}\right|>0$ for any $k_{n}$, where

$$
B_{k_{n}}=A_{k_{n}} \backslash A_{k_{n+1}}=\left\{t \in[0, \infty): 2^{k_{n+1}} \geq \frac{\lambda(t)}{\mu(t) K^{t}}>2^{k_{n}}\right\} ;
$$

otherwise, there will be $N \in \mathbb{N}$ such that for any $k \geq N$, we have $\left|B_{k}\right|=0$, and hence $\left|A_{k}\right|=0$ for any $k \geq N$, which is a contradiction. Since $\lambda \in L_{\text {loc }}^{1}$, we may also assume that $0<\int_{B_{k_{n}}} \lambda(t) d t<+\infty$ by replacing $B_{k_{n}}$ with a suitable bounded subset if necessary. Then we define $g$ by setting

$$
g(t)=\left\{\begin{array}{cc}
\frac{1}{\int_{B_{k_{n}}}^{\lambda(t) d t},}, & \forall t \in B_{k_{n}}, k_{n} \in \mathbb{N} ; \\
0, & \text { otherwise. }
\end{array}\right.
$$

It follows that

$$
\int_{0}^{+\infty} g(t) \lambda(t) d t=\sum_{k_{n} \in \mathbb{N}} \int_{B_{k_{n}}} \frac{\lambda(t)}{\int_{B_{k_{n}}} \lambda(t) d t} d t=\sum_{k_{n} \in \mathbb{N}} 1=+\infty
$$

and from the definition of $B_{k_{n}}$ that

$$
\begin{aligned}
\int_{0}^{+\infty} g(t) \mu(t) K^{t} d t & =\sum_{k_{n} \in \mathbb{N}} \int_{B_{k_{n}}} \frac{\mu(t) K^{t}}{\int_{B_{k_{n}}} \lambda(t) d t} d t \\
& \leq \sum_{k_{n} \in \mathbb{N}} 2^{-k_{n}} \int_{B_{k_{n}}} \frac{\lambda(t)}{\int_{B_{k_{n}}} \lambda(t) d t} d t=\sum_{k_{n} \in \mathbb{N}} 2^{-k_{n}}<\infty
\end{aligned}
$$

Hence Eq. 3.6 is satisfied.

For $p>1$, let $r(t)=\lambda(t)^{\frac{p}{p-1}} \mu(t)^{\frac{1}{1-p}} K^{\frac{t}{1-p}}$. Then we know that

$$
R_{p}=\int_{0}^{\infty} \lambda(t)^{\frac{p}{p-1}} \mu(t)^{\frac{1}{1-p}} K^{\frac{t}{1-p}} d t=\int_{0}^{\infty} r(t) d t=\infty .
$$

Since $\lambda^{p} / \mu \in L_{\mathrm{loc}}^{1 /(p-1)}([0, \infty))$, we have $r \in L_{\mathrm{loc}}^{1}([0, \infty))$. Define the function $g$ by setting

$$
g(t)=\lambda(t)^{\frac{1}{p-1}} \mu(t)^{\frac{1}{1-p}} K^{\frac{t}{1-p}} \alpha(t)=r(t) \alpha(t) / \lambda(t),
$$

where $\alpha:[0, \infty) \rightarrow[0, \infty]$ is be determined. Then to find a function $g$ satisfying Eq. 3.6, it suffices to show the existence of a function $\alpha$ satisfying

$$
\left\{\begin{array}{l}
\int_{0}^{+\infty} g(t) \lambda(t) d t=\int_{0}^{\infty} \alpha(t) r(t) d t=+\infty \\
\int_{0}^{+\infty} g(t)^{p} \mu(t) K^{t} d t=\int_{0}^{\infty} \alpha(t)^{p} r(t) d t<+\infty .
\end{array}\right.
$$

Consider the metric measure space $\left([0, \infty), d_{E}, \mu_{r}\right)$ with $d_{E}$ the Euclidean distance where $d \mu_{r}=r(t) d t$. Since $r \in L_{\mathrm{loc}}^{1}$, we have that $\left([0, \infty), d_{E}, \mu_{r}\right)$ is a $\sigma$-finite metric measure space. Then it follows from Eq. 3.7 that $\mu_{r}([0, \infty))=+\infty$. Hence by Lemma 3.4, we know that $L^{p}\left([0, \infty), \mu_{r}\right) \nsubseteq L^{1}\left([0, \infty), \mu_{r}\right)$, i.e., there exists a function $\alpha:[0, \infty) \rightarrow$ such that $\alpha \in L^{p}\left([0, \infty), \mu_{r}\right)$ but $\alpha \notin L^{1}\left([0, \infty), \mu_{r}\right)$. Choosing this $\alpha$ ensures Eq. 3.8.

In conclusion, for $1 \leq p<\infty$, we can construct a function $u \in \dot{N}^{1, p}(X)$ satisfying Eq. 3.5.

Remark 3.6 If additionally $\mu(X)<\infty$, instead of constructing the above increasing function, we may easily modify the construction so as to obtain a piecewise monotone function 
$u \in N^{1, p}(X)$ with values in $[0,1]$ so that $u(x)=1$ when $|x|=t_{2 j}$ and $u(x)=0$ when $|x|=t_{2 j+1}$, where $t_{k} \rightarrow \infty$ as $k \rightarrow \infty$. Then this oscillatory function $u$ belongs to $N^{1, p}(X)$, but has no limit along any geodesic ray. Hence we obtain the following result.

Proposition 3.7 Let $1 \leq p<\infty$ and assume that $R_{p}=+\infty$. If $\mu(X)<\infty$, then there exists a function $u \in N^{1, p}(X)$ such that $\lim _{[0, \xi) \ni x \rightarrow \xi} u(x)$ does not exist for any $\xi \in \partial X$.

Remark 3.8 Since our weights only depend on the distance to the root, Theorem 3.2 and Theorem 3.5 boil down to embeddings on the positive real axis. One of the key properties is that $R_{p}<\infty$ if and only if

$$
L^{p}\left(\mathbb{R}^{+}, K^{t} \mu(t) d t\right) \subset L^{1}\left(\mathbb{R}^{+}, \lambda(t) d t\right)
$$

where $\lambda$ and $\mu$ are defined on $[0, \infty)$ as specified in the introduction. Consequently, if $\mu(X)<\infty$, then $R_{p}<\infty$ implies $R_{q}<\infty$ whenever $1 \leq p<q<\infty$. However such an implication does not hold true if $\mu(X)=\infty$, but finiteness of $R_{p}$ is still subject to interpolation, i.e. if $R_{p}<\infty$ and $R_{r}<\infty$ then $R_{q}<\infty$ for every $q \in[p, r]$.

The above results give the full answers to the trace results for the homogeneous Newtonian space $\dot{N}^{1, p}(X)$ and also for the Newtonian space $N^{1, p}(X)$ when $\mu(X)<\infty$. We continue towards the case $\mu(X)=\infty$.

Proposition 3.9 Let $1 \leq p<\infty$ and assume $\mu(X)=\infty$. Then for every $f \in L^{p}(X)$, we have

$$
\liminf _{[0, \xi) \ni x \rightarrow \xi}|f(x)|=0, \quad \text { for a.e. } \xi \in \partial X,
$$

and hence $\operatorname{Tr} f=0$ if $\operatorname{Tr} f$ exists.

Proof Assume that Eq. 3.10 is false. Then there exist a function $f \in L^{p}(X)$ and a set $E \subset \partial X$ with $v(E)>0$ such that

$$
\liminf _{[0, \xi) \ni x \rightarrow \xi}|f(x)|>0, \text { for all } \xi \in E .
$$

Hence for each $\xi \in E$, there exist a constant $\epsilon(\xi)>0$ and an integer $N(\xi):=N(\epsilon(\xi))$ such that

$$
|f(x)| \geq \epsilon(\xi)>0, \quad \text { for all } x \in[0, \xi) \text { with }|x| \geq N(\xi) .
$$

It follows from Lemma 3.1 that

$$
\begin{aligned}
\|f\|_{L^{p}(X)}^{p} & =\int_{X}|f(x)|^{p} d \mu \approx \int_{\partial X} \int_{[0, \xi)}|f(x)|^{p} K^{j(x)} d \mu(x) d \nu(\xi) \\
& \geq \int_{E} \int_{\{x \in[0, \xi):|x| \geq N(\xi)\}}|f(x)|^{p} K^{j(x)} d \mu(x) d \nu(\xi) \\
& \geq \int_{E} \int_{\{x \in[0, \xi):|x| \geq N(\xi)\}} \epsilon(\xi)^{p} K^{j(x)} d \mu(x) d \nu(\xi) \\
& =\int_{E} \epsilon(\xi)^{p} \int_{N(\xi)}^{\infty} K^{j(t)} \mu(t) d t d \nu(\xi),
\end{aligned}
$$

where $j(t)$ is the largest integer such that $j(t) \leq t+1$. Since $\mu(X)=\infty$ and $\mu \in L_{\text {loc }}^{1}(X)$, for every integer $N(\xi)$, we have

$$
\int_{N(\xi)}^{\infty} K^{j(t)} \mu(t) d t=\infty .
$$


Since $\epsilon(\xi)>0$ for each $\xi \in E$ and $\nu(E)>0$, we obtain that

$$
\|f\|_{L^{p}(X)}^{p}=+\infty,
$$

which contradicts the fact that $f \in L^{p}(X)$. Thus Eq. 3.10 holds.

If $\operatorname{Tr} f$ exists, then $\operatorname{Tr}|f|$ also exists. It follows from the definition of the trace Eq. 1.1 and Eq. 3.10 that $\operatorname{Tr}|f|=0$. Hence $\operatorname{Tr} f=0$.

Proposition 3.10 Assume $R_{1}=+\infty$. Then there exists a function $u \in N^{1,1}(X)$ such that $\lim _{[0, \xi) \ni x \rightarrow \xi} u(x)$ does not exist, for any $\xi \in \partial X$.

Proof It follows from $R_{1}=\left\|\frac{\lambda(t)}{\mu(t) K^{t}}\right\|_{L^{\infty}([0, \infty))}=\infty$ that the sequence of sets

$$
E_{k}:=\left\{t \in[0, \infty): \frac{\lambda(t)}{\mu(t) K^{t}} \geq 2^{k}\right\}
$$

satisfies

$$
\left|E_{k}\right|>0 \text { for any } k \in \mathbb{N} \text {. }
$$

Hence we may choose a sequence $\left\{t_{k}: t_{k} \in[0, \infty)\right\}_{k \in \mathbb{N}}$ with

$$
t_{k} \rightarrow \infty \text { as } k \rightarrow \infty \text { and }\left|E_{k} \cap\left[t_{k-1}, t_{k}\right]\right|>0 \text { for any } k \in \mathbb{N} .
$$

Since $\mu \in L_{\text {loc }}^{1}([0, \infty))$, we have that for each $k \in \mathbb{N}$,

$$
0<\int_{t_{k-1}}^{t_{k}} \mu(t) K^{t} d t=: M_{k}<\infty .
$$

By the absolute continuity of integral with respect to measure, we may divide the interval $\left[t_{k-1}, t_{k}\right]$ into $\left\lceil 2^{k} M_{k}\right\rceil$ subintervals $\left\{I_{j}\right\}_{j}$ whose interiors are pairwise disjoint such that

$$
\bigcup_{j=1}^{\left\lceil 2^{k} M_{k}\right\rceil} I_{j}=\left[t_{k-1}, t_{k}\right] \text { and } 0<\int_{I_{j}} \mu(t) K^{t} d t \leq 2^{-k} .
$$

Since $\left|E_{k} \cap\left[t_{k-1}, t_{k}\right]\right|>0$ from Eq. 3.11, we obtain there is at least one subinterval $I_{k} \in$ $\left\{I_{j}\right\}_{j}$ such that $\left|E_{k} \cap I_{k}\right|>0$. Then we define a function $g$ by setting

$$
g(t)=\left\{\begin{array}{cc}
\frac{2}{\int_{E_{k} \cap I_{k}} \lambda(t) d t}, & \text { if } t \in E_{k} \cap I_{k}, k \in \mathbb{N} ; \\
0, & \text { otherwise. }
\end{array}\right.
$$

Since $\lambda(t)$ is always positive and $\lambda \in L_{\text {loc }}^{1}([0, \infty))$, the above definition is well-defined. Next we construct the function $u$. For any $k \in \mathbb{N}$, since we have

$$
\int_{t_{k-1}}^{t_{k}} g(t) \lambda(t) d t=\int_{E_{k} \cap I_{k}} \frac{2 \lambda(t)}{\int_{E_{k} \cap I_{k}} \lambda(t) d t} d t=2,
$$

we may apply the same idea of construction as in Remark 3.6 on $\left\{x \in X: t_{k-1} \leq|x| \leq t_{k}\right\}$ to obtain a piecewise monotone function $u$ with upper gradient $g_{u}(x)=g(|x|)$ and with values in $[0,1]$ so that $u(x)=0$ when $|x|=t_{k-1}, t_{k}$ and $u(x)=1$ when $|x|=t_{k}^{\prime}$ where $t_{k-1}<t_{k}^{\prime}<t_{k}$. Then the function $u$ has no limit along any geodesic ray. 
Thus it remains to show that $u \in N^{1,1}(X)$. We first estimate the $L^{1}$-norm of the upper gradient $g_{u}$ of $u$. By the definitions of function $g$ and of $E_{k}$, it follows from estimate Eq. 3.13 that

$$
\begin{aligned}
\int_{X} g_{u} d \mu & \lesssim \int_{0}^{\infty} g(t) \mu(t) K^{t} d t=\sum_{k \in \mathbb{N}} \int_{E_{k} \cap I_{k}} g(t) \mu(t) K^{t} d t \\
& \leq \sum_{k \in \mathbb{N}} 2^{-k} \int_{E_{k} \cap I_{k}} g(t) \lambda(t) d t=\sum_{k \in \mathbb{N}} 2^{1-k}<\infty .
\end{aligned}
$$

For the $L^{1}$-norm estimate of $u$, notice that $u(x)>0$ only if $|x| \in I_{k}$ for some $k \in \mathbb{N}$. Since $|u(x)| \leq 1$, we obtain from Eq. 3.12 that

$$
\int_{X} u d \mu=\sum_{k \in \mathbb{N}} \int_{\left\{x \in X:|x| \in I_{k}\right\}} u(x) d \mu(x) \lesssim \sum_{k \in \mathbb{N}} \int_{I_{k}} \mu(t) K^{t} d t \leq \sum_{k \in \mathbb{N}} 2^{-k}<\infty .
$$

We conclude that $u \in N^{1,1}(X)$ and that $\lim _{[0, \xi) \ni x \rightarrow \xi} u(x)$ does not exist, for any $\xi \in$ $\partial X$.

Lemma 3.11 Let $1<p<\infty$. Assume $\mu(X)=\infty$. The following are equivalent:

1. $\mathcal{R}_{p}<\infty$.

2. $\operatorname{Tr} f$ exists for every $f \in N^{1, p}(X)$ and $\operatorname{Tr} f(\xi) \equiv 0$ for a.e $\xi \in \partial X$.

3. $\operatorname{Tr} f$ exists for every $f \in N^{1, p}(X)$.

Proof 1. $\Rightarrow$ 2.: Let $f \in N^{1, p}(X)$. Lemma 3.1 gives that

$$
\int_{[0, \xi)}|f|^{p} K^{j(x)} d \mu<\infty \text { and } \int_{[0, \xi)} g_{f}^{p} K^{j(x)} d \mu<\infty
$$

for a.e. $\xi \in \partial X$. Let $\left\{A_{k}\right\}_{k=1}^{\infty}$ be a sequence of subintervals in $[0, \infty)$ with $\int_{A_{k}} K^{t} \mu(t) d t=$ 1 as in the formula Eq. 1.2 of $\mathcal{R}_{p}$. Then

$$
\int_{[0, \xi)}|f|^{p} K^{j(x)} d \mu=\sum_{k=1}^{\infty} \int_{A_{k}}|f(x(t))|^{p} K^{t} \mu(t) d t<\infty \text { for a.e. } \xi .
$$

Given $[0, \xi)$ satisfying the above inequality, we have

$$
\lim _{k \rightarrow \infty} \int_{A_{k}}|f(x(t))|^{p} K^{t} \mu(t) d t=0 .
$$

Since $f$ is continuous and $\int_{A_{k}} K^{t} \mu(t) d t=1$ for each $k$, there exists a $t_{k} \in \overline{A_{k}}$ such that

$$
\left|f\left(x_{t_{k}}\right)\right|^{p}=\int_{A_{k}}|f(x(t))|^{p} K^{t} \mu(t) d t \rightarrow 0 \text { as } \mathrm{k} \rightarrow \infty .
$$

Hence we have

$$
\begin{aligned}
\sup _{t \in A_{k}}|f(x(t))| & \leq\left|f\left(x\left(t_{k}\right)\right)\right|+\int_{A_{k}} g_{f} d s \\
& \leq\left|f\left(x\left(t_{k}\right)\right)\right|+\left(\int_{A_{k}} g_{f}^{p} K^{t} \mu(t) d t\right)^{1 / p}\left(\int_{A_{k}} \lambda^{\frac{p}{p-1}}(t) \mu^{\frac{1}{1-p}}(t) K^{\frac{t}{1-p}} d t\right)^{\frac{p-1}{p}} \\
& \rightarrow 0 \text { as } \mathrm{k} \rightarrow \infty,
\end{aligned}
$$


since $\mathcal{R}_{p}<\infty$. Since $\bigcup_{k=1}^{\infty} A_{k}=[0, \infty)$, it then follows that

$$
\left|\limsup _{[0, \xi) \ni x, x \rightarrow \xi} f(x)\right| \leq \lim _{k \rightarrow \infty} \sup _{t \in A_{k}}|f(x(t))|=0 \text { for a.e } \xi \in \partial X .
$$

Then $\operatorname{Tr} f$ exists and $\operatorname{Tr} f(\xi) \equiv 0$ for a.e $\xi \in \partial X$.

2. $\Rightarrow$ 3. This implication is trivial.

3. $\Rightarrow$ 1. Fix $p>1$, and suppose that $\mathcal{R}_{p}=\infty$. Then, for the sequence of subintervals $\left\{A_{k}\right\}_{k=1}^{\infty}$ with $\bigcup_{k=1}^{\infty} A_{k}=[0, \infty)$, we have

$$
\int_{A_{k}} K^{t} \mu(t) d t=1 \text { and } \sup _{k \in \mathbb{N}} \int_{A_{k}} \lambda^{\frac{p}{p-1}}(t) \mu^{\frac{1}{1-p}}(t) K^{\frac{t}{1-p}} d t=\infty .
$$

We pick a subsequence of $\left\{A_{k}\right\}_{k=1}^{\infty}$, still denoted $\left\{A_{k}\right\}_{k=1}^{\infty}$, such that

$$
\int_{A_{k}} K^{t} \mu(t) d t=1 \text { and } \int_{A_{k}} \lambda^{\frac{p}{p-1}}(t) \mu^{\frac{1}{1-p}}(t) K^{\frac{t}{1-p}} d t>4^{k} .
$$

Now, we divide $A_{k}$ into $2^{k}$ intervals $\left\{I_{k, l}\right\}_{l=1}^{2^{k}}$ with $\int_{I_{k, l}} K^{t} \mu(t) d t \equiv 2^{-k}$. Hence there exists at least one interval $I_{k}$ in $\left\{I_{k, l}: l=1,2, \ldots, 2^{k}\right\}$ such that

$$
\int_{I_{k}} K^{t} \mu(t) d t=2^{-k} \text { and } \int_{I_{k}} \lambda^{\frac{p}{p-1}}(t) \mu^{\frac{1}{1-p}}(t) K^{\frac{t}{1-p}} d t>2^{k} .
$$

We define

$$
g(t)=\sum_{k=1}^{\infty} \frac{\lambda^{\frac{1}{p-1}}(t) \mu^{\frac{1}{1-p}}(t) K^{\frac{t}{1-p}}}{\int_{I_{k}} \lambda^{\frac{p}{p-1}}(t) \mu^{\frac{1}{1-p}}(t) K^{\frac{t}{1-p}} d t} \chi_{I_{k}(t)}
$$

It follows that

$$
\begin{aligned}
\int_{0}^{\infty} g^{p}(t) K^{t} \mu(t) d t & =\sum_{k=1}^{\infty} \int_{I_{k}}\left(\frac{\lambda^{\frac{1}{p-1}}(t) \mu^{\frac{1}{1-p}}(t) K^{\frac{t}{1-p}}}{\int_{I_{k}} \lambda^{\frac{p}{p-1}}(t) \mu^{\frac{1}{1-p}}(t) K^{\frac{t}{1-p}} d t}\right)^{p} K^{t} \mu(t) d t \\
& =\sum_{k=1}^{\infty} \frac{1}{\left(\int_{I_{k}} \lambda^{\frac{p}{p-1}}(t) \mu^{\frac{1}{1-p}}(t) K^{\frac{t}{1-p}} d t\right)^{p-1}}<\sum_{k=1}^{\infty} \frac{1}{2^{k(p-1)}}<\infty
\end{aligned}
$$

and

$$
\int_{I_{k}} g(t) \lambda(t) d t=\int_{I_{k}} \frac{\lambda^{\frac{1}{p-1}}(t) \mu^{\frac{1}{1-p}}(t) K^{\frac{t}{1-p}}}{\int_{I_{k}} \lambda^{\frac{p}{p-1}}(t) \mu^{\frac{1}{1-p}}(t) K^{\frac{t}{1-p}} d t} \lambda(t) d t=1
$$

for every $I_{k}$ where $k=1,2, \ldots$. We use the procedure of Remark 3.6 by replacing the finiteness of the total measure with $\sum_{k} \mu\left(I_{k}\right)<\infty$ so as to obtain a piecewise monotone function $u \in N^{1, p}(X)$ with upper gradient $g_{u}(x)=g(|x|)$, where $g$ is from above, and so that $u$ has no limit along any geodesic ray. This contradicts 3 .

For $p=1$, we have $R_{1}=\mathcal{R}_{1}$. For $p>1$, it is easy to check that $R_{p}<\infty$ implies $\mathcal{R}_{p}<\infty$, while the inverse does not hold true. Furthermore, we will show that the finiteness of $\mathcal{R}_{p}$ will not imply the finiteness of $R_{q}$ for any $1 \leq q<\infty$.

For simplicity, we consider the special case where $\lambda$ and $\mu$ are piecewise constant. More precisely, assume that

$$
\lambda(t)=\lambda_{j}, \mu(t)=\mu_{j}, \text { for } t \in[j, j+1), j \in \mathbb{N},
$$


where $\left\{\lambda_{j}\right\}_{j \in \mathbb{N}}$ and $\left\{\mu_{j}\right\}_{j \in \mathbb{N}}$ are two sequences of positive and finite real numbers. Then

$$
d s=d \lambda(z)=\lambda_{j} d|z| \text { and } d \mu(z)=\mu_{j} d|z| \text {, for } j \leq|z|<j+1, j \in \mathbb{N} \text {. }
$$

We begin with easily checkable conditions.

Lemma 3.12 Let $1 \leq p<\infty$. Let $X$ be a $K$-regular tree with measure and metric as in Eq. 3.15. Then the following hold:

(i) $\mathcal{R}_{p}<\infty$ if

$$
\sup _{j}\left\{\max \left\{\frac{1}{K^{j} \mu_{j}}, \frac{\lambda_{j}^{p}}{K^{j} \mu_{j}}\right\}\right\}<+\infty .
$$

(ii) $\mathcal{R}_{p}=\infty$ if

$$
\sup _{j}\left\{\min \left\{\frac{1}{K^{j} \mu_{j}}, \frac{\lambda_{j}^{p}}{K^{j} \mu_{j}}\right\}\right\}=+\infty \text {. }
$$

Proof (i) If

$$
\sup _{j}\left\{\max \left\{\frac{1}{K^{j} \mu_{j}}, \frac{\lambda_{j}^{p}}{K^{j} \mu_{j}}\right\}\right\}<+\infty,
$$

there exists a constant $M>0$ such that

$$
\sup _{j}\left\{\frac{1}{K^{j} \mu_{j}}\right\} \leq M \text { and } \sup _{j}\left\{\frac{\lambda_{j}^{p}}{K^{j} \mu_{j}}\right\} \leq M .
$$

Let $A_{k}$ be as in formula Eq. 1.2 for $\mathcal{R}_{p}$. It is easy to see that

$$
\begin{aligned}
\mathcal{R}_{p} & =\sup _{k} \int_{A_{k}}\left(\frac{\lambda(t)^{p}}{K^{t} \mu(t)}\right)^{\frac{1}{p-1}} \frac{1}{K^{t} \mu(t)} K^{t} \mu(t) d t \\
& \lesssim\left(\sup _{j} \frac{\lambda_{j}^{p}}{K^{j} \mu_{j}}\right)^{\frac{1}{p-1}} \cdot \sup _{j} \frac{1}{K^{j} \mu_{j}} \cdot \sup _{k} \mu\left(A_{k}\right) \leq M \cdot M^{\frac{1}{p-1}}<\infty
\end{aligned}
$$

for $p>1$, and

$$
\mathcal{R}_{1}=R_{1}=\left\|\frac{\lambda(t)}{K^{t} \mu(t)}\right\|_{L^{\infty}([0, \infty))}=\sup _{j} \frac{\lambda_{j}}{K^{j} \mu_{j}} \leq M<\infty .
$$

(ii) If

$$
\sup _{j}\left\{\min \left\{\frac{1}{K^{j} \mu_{j}}, \frac{\lambda_{j}^{p}}{K^{j} \mu_{j}}\right\}\right\}=+\infty,
$$

there a subsequence $\left\{j_{n}: j_{n} \in \mathbb{N}\right\}$ such that

$$
\lim _{j_{n} \rightarrow \infty} \frac{1}{K^{j_{n}} \mu_{j_{n}}}=\infty \text { and } \lim _{j_{n} \rightarrow \infty} \frac{\lambda_{j_{n}}{ }^{p}}{K^{j_{n}} \mu_{j_{n}}}=\infty .
$$

Hence $\mu\left(\left[j_{n}, j_{n}+1\right]\right) \rightarrow 0$ as $j_{n} \rightarrow \infty$ and so we can assume that $\left[j_{n}, j_{n}+1\right] \subset A_{j_{n}}$ for $j_{n}$ big enough where $A_{j_{n}}$ belongs to $\left\{A_{k}\right\}_{k=1}^{\infty}$ as in Eq. 1.2. Thus

$$
\begin{aligned}
\mathcal{R}_{p} & \geq \sup _{j_{n}} \int_{\left[j_{n}, j_{n}+1\right]} \lambda^{\frac{p}{p-1}}(t) \mu^{\frac{1}{1-p}}(t) K^{\frac{t}{1-p}} d t \\
& =\sup _{j_{n}} \int_{\left[j_{n}, j_{n}+1\right]}\left(\frac{\lambda^{p}(t)}{K^{t} \mu(t)}\right)^{\frac{1}{p-1}} d t=\sup _{j_{n}}\left(\frac{\lambda_{j_{n}}^{p}}{K^{j_{n}} \mu_{j_{n}}}\right)^{\frac{1}{p-1}}=\infty
\end{aligned}
$$


for $p>1$, and

$$
\mathcal{R}_{1}=R_{1}=\left\|\frac{\lambda(t)}{K^{t} \mu(t)}\right\|_{L^{\infty}([0, \infty))}=\sup _{j} \frac{\lambda_{j}}{K^{j} \mu_{j}}=\infty .
$$

Remark 3.13 The conditions in Lemma 3.12 to determine whether $\mathcal{R}_{p}$ is finite or not are only sufficient conditions but not necessary ones. Towards this:

For (i), pick $\mu_{j}=K^{-j}(1+j)^{-1},\left(\lambda_{j}\right)^{p}=2^{-j}(1+j)^{-1}$. Then $\mathcal{R}_{p}<\infty$ but

$$
\sup _{j}\left\{\max \left\{\frac{1}{K^{j} \mu_{j}}, \frac{\lambda_{j}^{p}}{K^{j} \mu_{j}}\right\}\right\} \geq \sup _{j}\{1+j\}=\infty \text {. }
$$

For (ii), pick $\mu_{j}=K^{-j}, \lambda_{j}=2^{j}$. Then $\mathcal{R}_{p}=\infty$ but

$$
\sup _{j}\left\{\min \left\{\frac{1}{K^{j} \mu_{j}}, \frac{\lambda_{j}^{p}}{K^{j} \mu_{j}}\right\}\right\} \leq 1<+\infty \text {. }
$$

Example 3.14 Let $1<p<\infty$ and $1 \leq q<\infty$. We give simple examples where $\mathcal{R}_{p}<\infty$ but $R_{q}=\infty$.

(i) Let $\lambda_{j}=1$ and $\mu_{j}=K^{-j}$. Then $\mathcal{R}_{p}<\infty$ for every $1<p<\infty$, while $R_{q}=\infty$ for each $1<q<\infty$.

(ii) Let $1<p<\infty, \lambda_{j}=(1+j)^{-\alpha}$ and $\mu_{j}=K^{-j}(1+j)^{-\alpha-1}$ with $\alpha>p /(p-1)$. Then

$$
\mathcal{R}_{p} \leq R_{p} \leq \sum_{j=1}^{\infty} j^{\frac{\alpha+1-\alpha p}{p-1}}<\infty
$$

while $R_{1}=\sup _{j}\{j\}=\infty$.

Remark 3.15 The above examples show that $\mathcal{R}_{p}<\infty$ does not guarantee that $R_{q}<\infty$ for some $1 \leq q<\infty$ when $\mu(X)=\infty$ and $p>1$. Hence the existence of the trace $\operatorname{Tr}: N^{1, p} \rightarrow L_{v}^{p}(\partial X)$ is not equivalent to the finiteness of $R_{q}$ for some $1 \leq q<\infty$.

\section{Density}

In this section, we focus on the density properties of compactly supported functions in $N^{1, p}(X)$ and in $\dot{N}^{1, p}(X), 1 \leq p<\infty$. The function $\mathbf{1}$ is defined by $\mathbf{1}(x)=1$ for all $x$ in $X$ and we abuse the notation by using $\nabla u$ to denote $g_{u}$ if needed for convenience.

Our first result is an analog of the corresponding result for infinite networks [32], also see [24].

Lemma 4.1 Let $1 \leq p<\infty$ and assume that $\mu(X)<\infty$. Then we have that

$$
N_{0}^{1, p}(X)=N^{1, p}(X) \Longleftrightarrow \mathbf{1} \in N_{0}^{1, p}(X) .
$$

Proof Since it follows from $\mu(X)<\infty$ that $\mathbf{1} \in N^{1, p}(X)$, we obtain that $N_{0}^{1, p}(X)=$ $N^{1, p}(X)$ implies $\mathbf{1} \in N_{0}^{1, p}(X)$.

Towards the other direction, the hypothesis $\mathbf{1} \in N_{0}^{1, p}(X)$ gives a family of compactly supported functions $\left\{\mathbf{1}_{n}\right\}_{n \in \mathbb{N}}$ in $N^{1, p}(X)$ such that $\mathbf{1}_{n} \rightarrow \mathbf{1}$ in $N^{1, p}(X)$ as $n \rightarrow \infty$. Recall 
that $X^{m}:=\{x \in X:|x|<m\}$ for any $m \in \mathbb{N}$. Without loss of generality we may assume that $\mathbf{1}_{n}$ is nonnegative for any $n \in \mathbb{N}$ and that

$$
\left\|\mathbf{1}_{n}-\mathbf{1}\right\|_{N^{1, p}(X)}^{p}<\frac{1}{4^{p}} \mu\left(X^{1}\right), \text { for all } n \in \mathbb{N} .
$$

We claim that for any $n \in \mathbb{N}$, there exists a point $x_{n}$ with $x_{n} \in X^{1}$ such that $\left|1-\mathbf{1}_{n}\left(x_{n}\right)\right|<$ $1 / 4$. If not, then we have $\left|1-\mathbf{1}_{n}(x)\right| \geq \frac{1}{4}$ for any $x \in X^{1}$. Hence we obtain that

$$
\left\|1-\mathbf{1}_{n}\right\|_{N^{1, p}(X)}^{p} \geq\left\|1-\mathbf{1}_{n}\right\|_{N^{1, p}\left(X^{1}\right)}^{p} \geq \frac{1}{4^{p}} \mu\left(X^{1}\right),
$$

which is a contradiction. By the triangle inequality, we have $1-\mathbf{1}_{n}\left(x_{n}\right) \leq\left|1-\mathbf{1}_{n}\left(x_{n}\right)\right|<\frac{1}{4}$, and hence $\mathbf{1}_{n}\left(x_{n}\right)>\frac{3}{4}$.

Next, we claim that we may assume $\mathbf{1}_{n}(x)>1 / 2$ for all $x \in X^{n}$ by selecting a subsequence of $\left\{\mathbf{1}_{n}\right\}_{n \in \mathbb{N}}$ if necessary. Assume that this claim is not true. Then there exists $N \in \mathbb{N}$ such that for any $n \in \mathbb{N}$, there exists a point $y_{n} \in X^{N}$ with $\mathbf{1}_{n}\left(y_{n}\right) \leq 1 / 2$. Hence for any $n \in \mathbb{N}$, we have found two points $x_{n} \in X^{1}$ and $y_{n} \in X^{N}$ such that $\left|\mathbf{1}_{n}\left(x_{n}\right)-\mathbf{1}_{n}\left(y_{n}\right)\right| \geq 1 / 4$. Let $\gamma=\left[x_{n}, y_{n}\right]$ be the geodesic connecting $x_{n}$ and $y_{n}$. Then

$$
\int_{\gamma}\left|\nabla\left(\mathbf{1}_{n}\right)\right| d s \geq 1 / 4 \text { for any } n \in \mathbb{N} .
$$

By an argument similar to that for the estimate Eq. 2.1 and Eq. 2.2, we have that there exists a constant $C(N, p, \lambda, \mu)>0$ such that

$$
\int_{X}\left|\nabla\left(\mathbf{1}-\mathbf{1}_{n}\right)\right|^{p} d \mu=\int_{X}\left|\nabla\left(\mathbf{1}_{n}\right)\right|^{p} d \mu \geq C(N, p, \lambda, \mu)>0 \text { for any } n \in \mathbb{N},
$$

which is a contradiction to $\mathbf{1}_{n} \rightarrow \mathbf{1}$ in $N^{1, p}(X)$.

Thus, from the arguments above, we may assume that there exists a family of compactly supported functions $\left\{\mathbf{1}_{n}\right\}_{n \in \mathbb{N}}$ in $N^{1, p}(X)$ such that

$$
\left\{\begin{array}{l}
\mathbf{1}_{n} \rightarrow \mathbf{1} \text { in } N^{1, p}(X) \text { as } n \rightarrow \infty, \\
\mathbf{1}_{n}(x) \geq \frac{1}{2} \text { for any } x \in X^{n} .
\end{array}\right.
$$

We define $\overline{\mathbf{1}}_{n}:=\min \left\{2 \cdot \mathbf{1}_{n}, \mathbf{1}\right\}$ for all $n \in \mathbb{N}$. Then the family $\left(\overline{\mathbf{1}}_{n}\right)_{n \in \mathbb{N}}$ satisfies

$$
\left\{\begin{array}{l}
\overline{\mathbf{1}}_{n} \rightarrow \mathbf{1} \text { in } N^{1, p}(X) \text { as } n \rightarrow \infty, \\
\overline{\mathbf{1}}_{n} \equiv 1 \text { in } X^{n}, \\
\overline{\mathbf{1}}_{n} \text { is a function with compact support. }
\end{array}\right.
$$

Given a function $u$ in $N^{1, p}(X)$, let us show that $u_{n} \overline{\mathbf{1}}_{n} \rightarrow u$ in $N^{1, p}(X)$ where $u_{n}(x)$ is a truncation of $u$ with respect to $a_{n}:=\left\|\overline{\mathbf{1}}_{n}-\mathbf{1}\right\|_{N^{1, p}(X)}^{-1 / 2}$, namely

$$
u_{n}(x)=\left\{\begin{array}{ll}
\frac{u}{|u|} a_{n} & \text { if }|u| \geq a_{n} \\
u & \text { if }|u| \leq a_{n}
\end{array} .\right.
$$

From the basic properties of truncation (see for instance [11, Section 7.1]), we have that

$$
\left\{\begin{array}{l}
u_{n} \rightarrow u \text { in } N^{1, p}(X) \text { as } n \rightarrow \infty, \\
\left|u_{n}(x)\right| \leq a_{n}, \\
\left|\nabla u_{n}\right| \leq 3|\nabla u| .
\end{array}\right.
$$


We first show that $u_{n} \overline{\mathbf{1}}_{n} \rightarrow u$ in $L^{p}(X)$ as $n \rightarrow \infty$. By the triangle inequality, it follows from Eq. 4.1 and Eq. 4.2 that

$$
\begin{aligned}
\left\|u_{n} \overline{\mathbf{1}}_{n}-u\right\|_{L^{p}(X)} & \leq\left\|u_{n} \overline{\mathbf{1}}_{n}-u_{n}\right\|_{L^{p}(X)}+\left\|u_{n}-u\right\|_{L^{p}(X)} \\
& \leq a_{n}\left\|\overline{\mathbf{1}}_{n}-\mathbf{1}\right\|_{N^{1, p}(X)}+\left\|u_{n}-u\right\|_{L^{p}(X)} \\
& =\left\|\overline{\mathbf{1}}_{n}-\mathbf{1}\right\|_{N^{1, p}(X)}^{1 / 2}+\left\|u_{n}-u\right\|_{L^{p}(X)} \rightarrow 0 \text { as } n \rightarrow \infty .
\end{aligned}
$$

Recall that every function in $N^{1, p}(X)$ is locally absolutely continuous, see Section 2.2. By the product rule of locally absolutely continuous functions, we obtain that

$$
\begin{aligned}
\left|\nabla\left(u_{n} \overline{\mathbf{1}}_{n}-u\right)\right| & =\left|\nabla\left(u_{n} \overline{\mathbf{1}}_{n}-u_{n}+u_{n}-u\right)\right| \\
& \leq\left|u_{n}\right|\left|\nabla\left(\overline{\mathbf{1}}_{n}-\mathbf{1}\right)\right|+\left|\overline{\mathbf{1}}_{n}-\mathbf{1}\right|\left|\nabla u_{n}\right|+\left|\nabla\left(u_{n}-u\right)\right| \\
& \leq a_{n}\left|\nabla\left(\overline{\mathbf{1}}_{n}-\mathbf{1}\right)\right|+\left|\nabla u_{n}\right| \chi X \backslash X^{n}+\left|\nabla\left(u_{n}-u\right)\right| .
\end{aligned}
$$

Hence we obtain from the triangle inequality and Eq. 4.2 that

$$
\begin{aligned}
\left\|\nabla\left(u_{n} \overline{\mathbf{1}}_{n}-u\right)\right\|_{L^{p}(X)} & \leq a_{n}\left\|\nabla\left(\overline{\mathbf{1}}_{n}-\mathbf{1}\right)\right\|_{L^{p}(X)}+\left\|\nabla u_{n}\right\|_{L^{p}\left(X \backslash X^{n}\right)}+\left\|\nabla\left(u_{n}-u\right)\right\|_{L^{p}(X)} \\
& \leq\left\|\overline{\mathbf{1}}_{n}-\mathbf{1}\right\|_{N^{1, p}(X)}^{1 / 2}+3\|\nabla u\|_{L^{p}\left(X \backslash X^{n}\right)}+\left\|\nabla\left(u_{n}-u\right)\right\|_{L^{p}(X)},
\end{aligned}
$$

which tends to 0 as $n \rightarrow \infty$. Therefore, $u_{n} \overline{\mathbf{1}}_{n} \rightarrow u$ in $N^{1, p}(X)$ as $n \rightarrow \infty$. Since the support of $u_{n} \overline{\mathbf{1}}_{n}$ is compact, it follows from the definition of $N_{0}^{1, p}(X)$ that $u \in N_{0}^{1, p}(X)$, and hence $N_{0}^{1, p}(X)=N^{1, p}(X)$.

Notice that $\mathbf{1} \in \dot{N}^{1, p}(X)$ no matter if $\mu(X)$ is finite or not. By slightly modifying the previous proof, we obtain the following result.

Corollary 4.2 Let $1 \leq p<\infty$. Then the following statements are equivalent

$$
\dot{N}_{0}^{1, p}(X)=\dot{N}^{1, p}(X) \Longleftrightarrow \mathbf{1} \in \dot{N}_{0}^{1, p}(X)
$$

Applying Lemma 4.1, we obtain our first density result.

Proposition 4.3 Let $1 \leq p<\infty$ and assume that $\mu(X)<\infty$. Suppose additionally that $R_{p}=\infty$. Then we have that

$$
N_{0}^{1, p}(X)=N^{1, p}(X) .
$$

Proof It follows from Lemma 4.1 that it suffices to construct a sequence of compactly supported $N^{1, p}$-functions which converges to 1 in $N^{1, p}(X)$.

For $p>1$ and

$$
R_{p}=\int_{0}^{\infty} \lambda^{\frac{p}{p-1}}(t) \mu^{\frac{1}{1-p}}(t) K^{\frac{t}{1-p}} d t=\infty,
$$

we define the family of functions $\left\{\varphi_{n}\right\}_{n \in \mathbb{N}}$ as follows. For each $n \in \mathbb{N}$, let $r_{n}>n$ be an integer such that

$$
\int_{n}^{r_{n}} \lambda^{\frac{p}{p-1}}(t) \mu^{\frac{1}{1-p}}(t) K^{\frac{t}{1-p}} d t \geq 2^{n}
$$

We set $\varphi_{n}(x)=1$ for all $x \in X^{n}, \varphi_{n}(x)=0$ for all $x \in X \backslash X^{r_{n}}$ and

$$
\varphi_{n}(x)=1-\frac{\int_{n}^{|x|} \lambda^{\frac{p}{p-1}}(t) \mu^{\frac{1}{1-p}}(t) K^{\frac{t}{1-p}} d t}{\int_{n}^{r_{n}} \lambda^{\frac{p}{p-1}}(t) \mu^{\frac{1}{1-p}}(t) K^{\frac{t}{1-p}} d t}
$$


for all $x \in X^{r_{n}} \backslash X^{n}$. Since $\lambda^{p} / \mu \in L_{\text {loc }}^{1 /(p-1)}([0, \infty))$ and $\lambda, 1 / \mu>0$, then $\varphi_{n}$ is welldefined. It is easy to check that $\varphi_{n}$ is compactly supported.

By the construction of $\varphi_{n}$, an easy computation shows that

$$
\nabla\left(\varphi_{n}(x)-1\right)=0
$$

for all $x \in\left(X^{n}\right) \cup\left(X \backslash X^{r_{n}}\right)$ and that

$$
\left|\nabla\left(\varphi_{n}(x)-1\right)\right| \leq \frac{1}{\lambda(x)} \frac{\lambda^{\frac{p}{p-1}}(x) \mu^{\frac{1}{1-p}}(x) K^{\frac{|x|}{1-p}}}{\int_{n}^{r_{n}} \lambda^{\frac{p}{p-1}}(t) \mu^{\frac{1}{1-p}}(t) K^{\frac{t}{1-p}} d t}=\frac{\lambda^{\frac{1}{p-1}}(x) \mu^{\frac{1}{1-p}}(x) K^{\frac{|x|}{1-p}}}{\int_{n}^{r_{n}} \lambda^{\frac{p}{p-1}}(t) \mu^{\frac{1}{1-p}}(t) K^{\frac{t}{1-p}} d t}
$$

for all $x \in X^{r_{n}} \backslash X^{n}$.

Thanks to Eq. 4.4 and Eq. 4.5, we obtain the estimate

$$
\begin{aligned}
\int_{X}\left|\nabla\left(\varphi_{n}-\mathbf{1}\right)\right|^{p} d \mu & =\int_{X^{r_{n}} \backslash X^{n}}\left|\nabla\left(\varphi_{n}-\mathbf{1}\right)\right|^{p} d \mu \\
& \approx \int_{n}^{r_{n}} K^{t} \mu(t)\left(\frac{\lambda^{\frac{1}{p-1}}(t) \mu^{\frac{1}{1-p}}(t) K^{\frac{t}{1-p}}}{\int_{n}^{r_{n}} \lambda^{\frac{p}{p-1}}(t) \mu^{\frac{1}{1-p}}(t) K^{\frac{t}{1-p}} d t}\right)^{p} d t \\
& =\left(\int_{n}^{r_{n}} \lambda^{\frac{p}{p-1}}(t) \mu^{\frac{1}{1-p}}(t) K^{\frac{t}{1-p}} d t\right)^{1-p} .
\end{aligned}
$$

Since $p>1$ and Eq. 4.3 holds, we obtain that

$$
\left(\int_{n}^{r_{n}} \lambda^{\frac{p}{p-1}}(t) \mu^{\frac{1}{1-p}}(t) K^{\frac{1}{1-p}} d t\right)^{1-p} \rightarrow 0 \text { as } n \rightarrow \infty .
$$

Hence we have that $\left\|\nabla\left(\varphi_{n}-\mathbf{1}\right)\right\|_{L^{p}(X)} \rightarrow 0$ as $n \rightarrow \infty$. Moreover, since $\left|\varphi_{n}-\mathbf{1}\right| \leq 2 \chi_{X \backslash X^{n}}$, it follows from $\mu(X)<\infty$ that

$$
\left\|\varphi_{n}(x)-\mathbf{1}\right\|_{L^{p}(X)} \leq 2 \mu\left(X \backslash X^{n}\right) \rightarrow 0 \text { as } n \rightarrow \infty .
$$

Therefore, $\varphi_{n} \rightarrow \mathbf{1}$ in $N^{1, p}(X)$ as $n \rightarrow \infty$.

For $p=1$, since $\lambda / \mu \in L_{\mathrm{loc}}^{\infty}([0, \infty))$ implies that $\left\|\frac{\lambda(t)}{\mu(t) K^{t}}\right\|_{L^{\infty}([0, n))}<\infty$ for any $n \in \mathbb{N}$, it follows from $R_{1}=\left\|\frac{\lambda(t)}{\mu(t) K^{t}}\right\|_{L^{\infty}([0, \infty))}=\infty$ that the sequence of sets

$$
E_{k}:=\left\{t \in[k, \infty): \frac{\lambda(t)}{\mu(t) K^{t}} \geq 2^{k}\right\}, k \in \mathbb{N}
$$

is a nonincreasing sequence of subset of $[0, \infty)$ and that we have

$$
\left|E_{k}\right|>0 \text { for any } k \in \mathbb{N} \text {. }
$$

We have $E_{k}=\lim _{n \rightarrow \infty} E_{k} \cap[k, n]$ and $\left|E_{k}\right|=\lim _{n \rightarrow \infty}\left|E_{k} \cap[k, n]\right|$. Hence there exist a $k_{n}>k$ such that

$$
E_{k_{n}}:=\left\{t \in\left[k, k_{n}\right]: \frac{\lambda(t)}{\mu(t) K^{t}} \geq 2^{k}\right\}
$$

satisfies $0<\left|E_{k_{n}}\right|<\infty$.

We define a sequence $\left\{\varphi_{k}\right\}$ of functions by setting

$$
\varphi_{k}(x)=1-\frac{1}{\left|E_{k_{n}}\right|} \int_{k}^{|x|} \chi_{E_{k_{n}}}(t) d t
$$

for all $|x| \in\left[k, k_{n}\right]$ and $\varphi_{k}(x)=0$ on $X \backslash X^{k_{n}}, \varphi_{k}(x)=1$ on $X^{k}$. 
It follows directly from the definition of $\varphi_{k}$ that each $\varphi_{k}$ has compact support and that

$$
\left.\left|\varphi_{k}-1\right| \leq 2 \chi_{X \backslash X^{k}}, \quad \mid \nabla\left(\varphi_{k}\right)(x)-1\right) \mid \leq \frac{\chi_{E_{k_{n}}}(x)}{\lambda(x)\left|E_{k_{n}}\right|} .
$$

Hence, thanks to $\mu(X)<\infty$ and the definition of $E_{k_{n}}$, we obtain that

$$
\begin{aligned}
\left\|\varphi_{k}-1\right\|_{N^{1,1}(X)} & =\left\|\varphi_{k}-1\right\|_{L^{1}(X)}+\left\|\nabla\left(\varphi_{k}-1\right)\right\|_{L^{1}(X)} \\
& \leq\left\|2 \chi_{X \backslash X^{k}}\right\|_{L^{1}(X)}+\int_{X} \frac{\chi_{E_{k_{n}}}(t)}{\lambda(t)\left|E_{k_{n}}\right|} d \mu(t) \\
& \lesssim 2 \mu\left(X \backslash X^{k}\right)+\frac{1}{\left|E_{k_{n}}\right|} \int_{k}^{k_{n}} \frac{\mu(t) K^{t}}{\lambda(t)} \chi_{E_{k_{n}}}(t) d t \\
& \leq 2 \mu\left(X \backslash X^{k}\right)+\frac{1}{2^{k}} \rightarrow 0 \text { as } k \rightarrow \infty .
\end{aligned}
$$

Hence $\varphi_{k} \rightarrow \mathbf{1}$ in $N^{1,1}(X)$ as $k \rightarrow \infty$.

By using the same construction of the sequence of compactly supported $N^{1, p}$-functions as the one in the above proof, we obtain the following corollary immediately from Corollary 4.2.

Corollary 4.4 Let $1 \leq p<\infty$. Assume $R_{p}=\infty$. Then we obtain that

$$
\dot{N}_{0}^{1, p}(X)=\dot{N}^{1, p}(X) .
$$

Proposition 4.5 Let $1 \leq p<\infty$ and assume that $\mu(X)<\infty$. Suppose additionally that $R_{p}<\infty$. Then we have

$$
N_{0}^{1, p}(X) \subsetneq N^{1, p}(X)
$$

Proof Suppose $N_{0}^{1, p}(X)=N^{1, p}(X)$. Since $1 \in N^{1, p}(X)$, it follows that for every $\varepsilon>0$, there exists a function $u \in N^{1, p}(X)$ with compact support such that

$$
\|1-u\|_{N^{1, p}(X)}<\varepsilon
$$

Let $\xi \in \partial X$ be arbitrary, and $x_{j}:=x_{j}(\xi)$ be the ancestor of $\xi$ with $\left|x_{j}\right|=j$ and $x_{0}=0$. Let $0<\epsilon<\frac{1}{2}\|\mu\|_{L^{1}([0,1])}^{1 / p}$. By repeating the argument in the beginning of Proof of Lemma 4.1 with the change that we replace $\mu\left(X^{1}\right) / 4^{p}$ and $X^{1}$ by $\epsilon^{p}$ and $\left[0, x_{1}(\xi)\right]$, respectively, we obtain the existence of $x_{\xi} \in\left[0, x_{1}(\xi)\right]$ for which the function $u$ in Eq. 4.6 satisfies $\left|1-u\left(x_{\xi}\right)\right|<\frac{1}{2}$. By the triangle inequality, we have $1-\left|u\left(x_{\xi}\right)\right| \leq\left|1-u\left(x_{\xi}\right)\right|<\frac{1}{2}$, and hence $\left|u\left(x_{\xi}\right)\right|>\frac{1}{2}$.

Notice that $u$ has compact support. Then for any $\xi \in \partial X$, we have $\lim _{n \rightarrow \infty} u\left(x_{n}(\xi)\right)=0$ and that

$$
\frac{1}{2}<\lim _{n \rightarrow \infty}\left|u\left(x_{\xi}\right)-u\left(x_{n}(\xi)\right)\right| \leq \int_{[0, \xi)} g_{u} d s=\int_{[0, \xi)} g_{u} \frac{\lambda(x)}{\mu(x)} d \mu .
$$


For $p=1$, integrating over all $\xi \in \partial X$, since $v(\partial X)=1$, we obtain by Fubini's theorem that

$$
\begin{aligned}
\frac{1}{2} & \leq \int_{\partial X} \int_{X} g_{u}(x) \chi_{[0, \xi)}(x) \frac{\lambda(x)}{\mu(x)} d \mu(x) d v(\xi) \\
& =\int_{X} g_{u}(x) \frac{\lambda(x)}{\mu(x)}\left(\int_{\partial X} \chi_{[0, \xi)}(x) d \nu(\xi)\right) d \mu(x) \\
& =\int_{X} g_{u}(x) \lambda(x) \mu(x)^{-1} v\left(I_{x}\right) d \mu(x),
\end{aligned}
$$

where $I_{x}=\{\xi \in \partial X: x<\xi\}$. Since $v\left(I_{x}\right) \approx K^{-|x|}$, we obtain from $R_{1}<\infty$ that

$$
\frac{1}{2} \lesssim R_{1} \int_{X} g_{u}(x) d \mu(x) \lesssim\|1-u\|_{N^{1,1}(X)}<\epsilon .
$$

By choosing $\epsilon$ small enough, the above estimate yields a contradiction, and hence $N_{0}^{1,1}(X) \neq N^{1,1}(X)$.

For $p>1$, by Eq. 4.7 and the Hölder inequality, we have that

$$
\begin{aligned}
\frac{1}{2^{p}} & \leq\left(\int_{[0, \xi)} g_{u} K^{j(x) / p} \frac{\lambda(x)}{\mu(x) K^{j(x) / p}} d \mu\right)^{p} \\
& \leq \int_{[0, \xi)} g_{u}^{p} K^{j(x)} d \mu\left(\int_{[0, \xi)}\left(\frac{\lambda(x)}{\mu(x) K^{j(x) / p}}\right)^{\frac{p}{p-1}} d \mu\right)^{p-1} \\
& \lesssim R_{p}^{p-1} \int_{[0, \xi)} g_{u}^{p} K^{j(x)} d \mu,
\end{aligned}
$$

where $j(x)$ is the largest integer such that $j(x) \leq|x|+1$. Here the last inequality holds since

$$
\int_{[0, \xi)}\left(\frac{\lambda(x)}{\mu(x) K^{j(x) / p}}\right)^{\frac{p}{p-1}} d \mu \approx \int_{0}^{\infty} \frac{\lambda(t)^{\frac{p}{p-1}}}{\mu(t)^{\frac{p}{p-1}} K^{\frac{t}{p-1}}} \mu(t) d t=R_{p} .
$$

Integrating over all $\xi \in \partial X$, since $\nu(\partial X)=1$ and $R_{p}<+\infty$, we obtain by Fubini's theorem that

$$
\begin{aligned}
\frac{1}{2^{p}} & \lesssim \int_{\partial X} \int_{X} g_{u}(x)^{p} \chi_{[0, \xi)}(x) K^{j(x)} d \mu(x) d \nu(\xi) \\
& =\int_{X} g_{u}(x)^{p} K^{j(x)}\left(\int_{\partial X} \chi_{[0, \xi)}(x) d \nu(\xi)\right) d \mu(x) \\
& =\int_{X} g_{u}(x)^{p} K^{j(x)} \nu\left(I_{x}\right) d \mu(x),
\end{aligned}
$$

where the notations $I_{x}$ and $j(x)$ are the same ones as those we used before. Since $v\left(I_{x}\right) \approx$ $K^{-j(x)}$, we obtain the estimate

$$
\frac{1}{2} \lesssim\left(\int_{X} g_{u}(x)^{p} d \mu(x)\right)^{1 / p} \leq\|1-u\|_{N^{1, p}(X)}<\epsilon .
$$

By choosing $\epsilon$ small enough, the above estimate gives a contradiction, and hence $N_{0}^{1, p}(X) \neq N^{1, p}(X)$ for $p>1$. 1.

Since $N_{0}^{1, p}(X) \subset N^{1, p}(X)$ by definition, we obtain $N_{0}^{1, p}(X) \subsetneq N^{1, p}(X)$ for all $p \geq$ 
Corollary 4.6 Let $p \geq 1$ and assume that $R_{p}<\infty$. Then we have

$$
\dot{N}_{0}^{1, p}(X) \subsetneq \dot{N}^{1, p}(X) .
$$

Proof Suppose $\dot{N}_{0}^{1, p}(X)=\dot{N}^{1, p}(X)$. Since $1 \in \dot{N}^{1, p}(X)$, it follows that for every $\epsilon>0$, there exists a function $u \in \dot{N}^{1, p}(X)$ with compact support such that

$$
\|1-u\|_{\dot{N}^{1, p}(X)}<\epsilon .
$$

Then by the definition of our $\dot{N}^{1, p}$-norm, we have $|u(0)-1|<\epsilon$ and hence $|u(0)|>1-\epsilon$.

Then using a similar argument to the one in the Proof of Proposition 4.5 (replace $u\left(x_{\xi}\right)$ with $u(0))$, we obtain a contradiction. The claim follows.

The above results give a full picture for the density properties for homogeneous Newtonian spaces $\dot{N}^{1, p}(X)$ and for Newtonian spaces $N^{1, p}(X)$ when $\mu(X)<\infty$. When the total measure is infinite, the density results for the Newtonian space $N^{1, p}(X)$ are quite different.

Lemma 4.7 Let $K=1$, i.e., $X$ be a l-regular tree and assume that $\mu(X)=\infty$. Then for any $f \in N^{1, p}(X)$, there exists a sequence of compactly supported $N^{1, p}$-functions $\left\{f_{n}\right\}_{n \in \mathbb{N}}$ such that $f_{n} \rightarrow f$ in $N^{1, p}(X)$.

Proof Notice that we may compose any $f \in N^{1, p}(X)$ as $f=f^{+}-f^{-}$where $f^{+}=$ $f \cdot \chi_{\{f \geq 0\}} \geq 0$ and $f^{-}=-f \cdot \chi_{\{f \leq 0\}} \geq 0$. Hence we may assume that $f \geq 0$.

Since $K=1, \partial X$ contains only one point $\xi_{0}$ and there is a unique geodesic ray. It follows from Proposition 3.9 that

$$
\liminf _{\left[0, \xi_{0}\right) \ni x \rightarrow \xi_{0}} f(x)=0 .
$$

Denote by $x_{n}$ the vertex of $X$ with $\left|x_{n}\right|=n$ when $n \in \mathbb{N}$. Then it follows from Eq. 4.8 that

$$
f\left(x_{n}\right)-\int_{\left[x_{n}, \xi_{0}\right)} g_{f} d s \leq 0, \forall n \in \mathbb{N} .
$$

We define functions $f_{n}$ by setting

$$
f_{n}(x):= \begin{cases}f(x), & \text { if }|x| \leq n \\ \max \left\{0, f\left(x_{n}\right)-2 \int_{\left[x_{n}, x\right]} g_{f} d s\right\}, & \text { if }|x|>n .\end{cases}
$$

Then it is easy to check that $f_{n} \in N^{1, p}(X)$, since $0 \leq f_{n} \leq f$ and $g_{f_{n}} \leq 2 g_{f}$. Next, we check that $f_{n}$ is compactly supported. Assume not. Since $f_{n}$ is non-increasing for $|x|>n$ by definition, we have that $f_{n}(x)>0$ for any $|x|>n$ and hence that

$$
\lim _{x \rightarrow \xi_{0}} f_{n}(x)=f\left(x_{n}\right)-2 \int_{\left[x_{n}, \xi_{0}\right)} g_{f} d s \geq 0 .
$$

Combining this with Eq. 4.9, we conclude that

$$
\int_{\left[x_{n}, \xi_{0}\right)} g_{f} d s=0
$$

Then $g_{f}=0$ for $|x|>n$ and it follows from Eq. 4.8 that $f$ and $f_{n}$ has to be identically 0 for $|x| \geq n$, which is a contradiction. Hence $f_{n}$ is compactly supported. 
At last, we estimate the $N^{1, p}$-norm of $f_{n}-f$. By the fact that $0 \leq f_{n} \leq f$ and $g_{f_{n}} \leq$ $2 g_{f}$, we obtain the estimate

$$
\begin{aligned}
\left\|f_{n}-f\right\|_{N^{1, p}(X)} & =\left\|f_{n}-f\right\|_{N^{1, p}(X \cap\{|x| \geq n\})} \\
& \leq\left\|f_{n}\right\|_{N^{1, p}(X \cap\{|x| \geq n\})}+\|f\|_{N^{1, p}(X \cap\{|x| \geq n\})} \\
& \leq 3\|f\|_{N^{1, p}(X \cap\{|x| \geq n\})} \rightarrow 0 \text { as } n \rightarrow 0,
\end{aligned}
$$

since $f \in N^{1, p}(X)$. Thus $\left\{f_{n}\right\}_{n \in \mathbb{N}}$ is a sequence of compactly supported $N^{1, p}$-functions with $f_{n} \rightarrow f$ in $N^{1, p}(X)$, which finishes the proof.

If $\int_{0}^{\infty} \lambda(t) d t=\infty$, then $X$ is complete and unbounded with respect to distance $d$ and it follows by using suitable cutoff functions that $N_{0}^{1, p}(X)=N^{1, p}(X)$. Our next result shows that this is also the case when $X$ is bounded and not complete if we assume $\mu(X)=\infty$.

Theorem 4.8 Let $1 \leq p<\infty$ and assume that $\mu(X)=\infty$. Then we have that

$$
N_{0}^{1, p}(X)=N^{1, p}(X) .
$$

Proof If $K=1$, the result follows directly from Lemma 4.7. Hence we assume $K \geq 2$ in the ensuing proof.

For any $f \in N^{1, p}(X)$, by the same argument as in Lemma 4.7, we may assume that $f \geq 0$. It suffices to construct a sequence $\left\{f_{n}\right\}_{n \in \mathbb{N}}$ of compactly supported $N^{1, p}$-functions such that $f_{n} \rightarrow f$ in $N^{1, p}(X)$.

For each $n \in \mathbb{N}$, we denote by $\left\{x_{n, j}\right\}_{j=1}^{K^{n}}$ the vertices of $n$-level, i.e., $\left|x_{n, j}\right|=n$ for all $j=1, \cdots, K^{n}$. For any $x_{n, j}$, we study the subtree $\Gamma_{x_{n, j}}$ which is a subset of $X$ with root $x_{n, j}$. More precisely,

$$
\Gamma_{x_{n, j}}:=\left\{x \in X: x_{n, j}<x\right\} .
$$

Since every vertex has exactly $K$ children, we may divide $\Gamma_{x_{n, j}}$ into $K$ subsets, where each subset contains a subtree whose root is a child of $x_{n, j}$ and an edge connecting this child with $x_{n, j}$. We denote by $\left\{\Gamma_{x_{n, j}}^{i}\right\}_{i=1}^{K}$ these $K$ subsets.

Fix $f \in N^{1, p}(X)$. We first study the function $u:=\left.f\right|_{\Gamma_{x_{n, j}}}$. If $\|f\|_{N^{1, p}\left(\Gamma_{x_{n, j}}\right)}>0$, we first modify the function $u$ to a function $v$ with $v(x)=v(|x|)$ for any $x \in \Gamma_{x_{n, j}}$, i.e., for any $x, y \in \Gamma_{x_{n, j}}$ with $|x|=|y|$, then $v(x)=v(y)$. The modification procedure is as follows:

Step 1 Since $\Gamma_{x_{n, j}}=\bigcup_{i=1}^{K} \Gamma_{x_{n, j}}^{i}$, without loss of generality, we may assume

$$
\|u\|_{N^{1, p}\left(\Gamma_{x_{n, j}}^{1}\right)}=\min \left\{\|u\|_{N^{1, p}\left(\Gamma_{x_{n, j}}^{i}\right)}: i=1,2, \ldots, K\right\} .
$$

Then we define a function $u^{1}$ by identically copying the minimal $N^{1, p}$-energy subtree of $u$ (here is $\left.u\right|_{\Gamma_{x_{n, j}}^{1}}$ ), to the other $k-1$ subtrees $\Gamma_{x_{n, j}}^{i}, i=2, \cdots K$. More precisely,

$$
u^{1}(x):= \begin{cases}u(x), & \text { if } x \in \Gamma_{x_{n, j}}^{1} \\ \left.u\right|_{\Gamma_{x_{n, j}}^{1}}(y) \text { with } y \in \Gamma_{x_{n, j}}^{1},|y|=|x|, & \text { if } x \in \Gamma_{x_{n, j}}^{i}\end{cases}
$$

It follows from Eq. 4.10 that

$$
\left\|u^{1}\right\|_{N^{1, p}\left(\Gamma_{x_{n, j}}\right)} \leq\|u\|_{N^{1, p}\left(\Gamma_{x_{n, j}}\right)} .
$$

Then for any $x, y \in \Gamma_{x_{n, j}} \cap\{x \in X: n \leq|x| \leq n+1\}$ with $|x|=|y|$, we have $u^{1}(x)=u^{1}(y)$. 
Step 2 Denote by $\left\{x_{n+1, t}\right\}_{t=1}^{K}$ the $K$ children of $x_{n, j}$. We repeat the Step 1 by replacing the function $u$ and $\Gamma_{x_{n, j}}$ with $u^{1}$ and $\Gamma_{x_{n+1, t}}$, respectively. Here we repeat the Step 1 for all $K$ subtrees $\Gamma_{x_{n+1, t}}, t=1, \cdots, K$. Hence we obtain a function $u^{2}$ on $\Gamma_{x_{n, j}}$ by additionally letting $u^{2}(x)=u^{1}(x)$ if $x \in \Gamma_{x_{n, j}}$ with $n \leq|x| \leq n+1$. Moreover, it is easy to check that

$$
\left\|u^{2}\right\|_{N^{1, p}\left(\Gamma_{x_{n, j}}\right)} \leq\left\|u^{1}\right\|_{N^{1, p}\left(\Gamma_{x_{n, j}}\right)} \leq\|u\|_{N^{1, p}\left(\Gamma_{x_{n, j}}\right)}
$$

and that $u^{2}(x)=u^{2}(y)$ for any $x, y \in \Gamma_{x_{n, j}} \cap\{x \in X: n \leq|x| \leq n+2\}$ with $|x|=|y|$.

Continuing this procedure, we obtain a sequence of functions $\left\{u^{k}\right\}_{k \in \mathbb{N}}$. We define $v=$ $\lim _{k \rightarrow \infty} u^{k}$. Then we know from induction that

$$
\|v\|_{N^{1, p}\left(\Gamma_{x_{n, j}}\right)} \leq\|u\|_{N^{1, p}\left(\Gamma_{\left.x_{n, j}\right)}\right.}=\|f\|_{N^{1, p}\left(\Gamma_{x_{n, j}}\right)}
$$

and that $v(x)=v(y)$ for any $x, y \in \Gamma_{x_{n, j}}$ with $|x|=|y|$.

The value of function $v(x)$ only depends on the distance $d\left(x_{n, j}, x\right)$. We may regard $v$ as a function on a 1-regular tree with root $x_{n, j}$ and infinite measure, since $\mu\left(\Gamma_{x_{n, j}}\right)=\infty$. Hence, from the Proof of Lemma 4.7, we are able to choose a compactly supported $N^{1, p}$-function $f_{n, j}$ on $\Gamma_{x_{n, j}}$ with

$$
\left\|f_{n, j}-v\right\|_{N^{1, p}\left(\Gamma_{\left.x_{n, j}\right)}\right.} \leq 3\|u\|_{N^{1, p}\left(\Gamma_{\left.x_{n, j}\right)}\right.}=3\|f\|_{N^{1, p}\left(\Gamma_{x_{n, j}}\right)} .
$$

Then it follows from Eq. 4.11 and Eq. 4.12 that

$$
\begin{aligned}
\left\|f_{n, j}-f\right\|_{N^{1, p}\left(\Gamma_{x_{n, j}}\right)} & \leq\left\|f_{n, j}-v\right\|_{N^{1, p}\left(\Gamma_{x_{n, j}}\right)}+\|v-f\|_{N^{1, p}\left(\Gamma_{x_{n, j}}\right)} \\
& \leq\left\|f_{n, j}-v\right\|_{N^{1, p}\left(\Gamma_{\left.x_{n, j}\right)}\right.}+\|v\|_{N^{1, p}\left(\Gamma_{x_{n, j}}\right)}+\|f\|_{N^{1, p}\left(\Gamma_{x_{n, j}}\right)} \\
& \leq 5\|f\|_{N^{1, p}\left(\Gamma_{\left.x_{n, j}\right)} .\right.}
\end{aligned}
$$

If $\|f\|_{N^{1, p}\left(\Gamma_{\left.x_{n, j}\right)}\right.}=0$, then $f=0$ on $\Gamma_{x_{n, j}}$ and we just define $f_{x_{n, j}}=\left.f\right|_{\Gamma_{x_{n, j}}}$.

At last, we define a function $f_{n}$ by setting

$$
f_{n}(x):= \begin{cases}f(x), & \text { if }|x| \leq n \\ f_{n, j}(x), & \text { if } x \in \Gamma_{x_{n, j}}\end{cases}
$$

Then it is easy to check that $f_{n} \in N^{1, p}(X)$ and that $f_{n}$ is compactly supported, since $f_{n, j}$ are compactly supported for any $j=1, \cdots, K^{n}$. It follows from estimate Eq. 4.13 that

$$
\begin{aligned}
\left\|f_{n}-f\right\|_{N^{1, p}(X)} & =\left\|f_{n}-f\right\|_{N^{1, p}(X \cap\{|x| \geq n\})}=\sum_{j=1}^{K^{n}}\left\|f_{n}-f\right\|_{N^{1, p}\left(\Gamma_{x_{n, j}}\right)} \\
& =\sum_{j=1}^{K^{n}}\left\|f_{n, j}-f\right\|_{N^{1, p}\left(\Gamma_{x_{n, j}}\right)} \leq 5 \sum_{j=1}^{K^{n}}\|f\|_{N^{1, p}\left(\Gamma_{x_{n, j}}\right)} \\
& =5\|f\|_{N^{1, p}(X \cap\{|x| \geq n\})} \rightarrow 0, \text { as } n \rightarrow 0,
\end{aligned}
$$

since $f \in N^{1, p}(X)$. Thus we have found a sequence $\left\{f_{n}\right\}_{n \in \mathbb{N}}$ of compactly supported $N^{1, p}$ functions with $f_{n} \rightarrow f$ in $N^{1, p}(X)$, which finishes the proof. 


\section{Proofs of Theorems}

Proof of Theorem 1.1 (i) $\Rightarrow$ (ii) is given by Corollary 3.3; (ii) $\Rightarrow$ (iii) is trivial and (iii) $\Rightarrow($ i $)$ is given by Proposition 3.7.

$(i) \Rightarrow(i v)$ is given by Proposition 4.5 and $(i v) \Rightarrow(i)$ is given by Corollary 4.3.

Proof of Theorem 1.2 Part (1) is given by Theorem 4.8.

Part (2): For $p>1$, the claim is given by Lemma 3.11. For $p=1$, the claim follows by Corollary 3.3 and Proposition 3.10.

Proof of Theorem 1.3 (i) $\Rightarrow$ (ii) is given by Theorem 3.2; (ii) $\Rightarrow$ (iii) is trivial and (iii) $\Rightarrow$ (i) is given by Theorem 3.5 .

$(i) \Rightarrow(i v)$ is given by Corollary 4.6 and $(i v) \Rightarrow(i)$ is given by Corollary 4.4 .

Acknowledgment The authors would like to thank the referees for their useful comments that especially allowed as to improve on the original versions of Proposition 2.2 and Lemma 3.11.

Funding Open access funding provided by University of Jyväskylä (JYU).

Open Access This article is licensed under a Creative Commons Attribution 4.0 International License, which permits use, sharing, adaptation, distribution and reproduction in any medium or format, as long as you give appropriate credit to the original author(s) and the source, provide a link to the Creative Commons licence, and indicate if changes were made. The images or other third party material in this article are included in the article's Creative Commons licence, unless indicated otherwise in a credit line to the material. If material is not included in the article's Creative Commons licence and your intended use is not permitted by statutory regulation or exceeds the permitted use, you will need to obtain permission directly from the copyright holder. To view a copy of this licence, visit http://creativecommonshorg/licenses/by/4.0/.

\section{References}

1. Aronszajn, N.: Boundary values of functions with finite Dirichlet integral, Techn. Report 14 University of Kansas (1955)

2. Björn, A., Björn, J.: Nonlinear potential theory on metric spaces, EMS Tracts Math. 17, European Mathematical Society, Zürich. xii+403 pp (2011)

3. Björn, A., Björn, J., Gill, J.T., Shanmugalingam, N.: Geometric analysis on Cantor sets and trees. J. Reine Angew. Math. 725, 63-114 (2017)

4. Björn, A., Björn, J.: N Shanmugalingam, Bounded geometry and $p$-harmonic functions under uniformization and hyperbolization. Accepted by J. Geom. Anal. https://doi.org/10.1007/s12220-02000477-0

5. Falconer, K.: Techniques in Fractal Geometry. Wiley, Chichester (1997)

6. Gagliardo, E.: Caratterizzazioni delle tracce sulla frontiera relative ad alcune classi di funzioni in $n$ variabili. Rend. Sem. Mat. Univ. Padova 27, 284-305 (1957)

7. Grigor'yan, A.: Analytic and geometric background of recurrence and non-explosion of the Brownian motion on Riemannian manifolds. Bull. Amer. Math. Soc. (N.S.) 36(2), 135-249 (1999)

8. Hajłasz, P.: Sobolev space on metric-measure spaces, in Heat kernels and analysis on manifolds, graphs and metric spaces (Paris 2002). Contemp. Math. 338, 173-218 (2003). American mathematical society, Providence

9. Hajłasz, P., Martio, O.: Traces of Sobolev functions on fractal type sets and characterization of extension domains. J. Funct. Anal. 143(1), 221-246 (1997)

10. Heinonen, J., Koskela, P.: Quasiconformal mappings in metric spaces with controlled geometry. Acta Math. 181, 1-61 (1998)

11. Heinonen, J., Koskela, P., Shanmugalingam, N., Tyson, J.: Sobolev spaces on metric measure spaces: an approach based on upper gradients cambridge: cambridge university press (2015) 
12. Holopainen, I.: Nonlinear potential theory and quasiregular mappings on Riemannian manifolds. Ann. Acad. Sci. Fenn. Ser. A I Math Dissertationes 74, 45 (1990)

13. Holopainen, I.: Rough isometries and $p$-harmonic functions with finite Dirichlet integral. Rev. Mat. Iberoamericana 10(1), 143-176 (1994)

14. Ihnatsyeva, L., Vähäkangas, A.: Characterization of traces of smooth functions on Ahlfors regular sets. J. Funct. Anal. 265(9), 1870-1915 (2013)

15. Korte, R., Lahti, P., Li, X., Shanmugalingam, N.: Notions of Dirichlet problem for functions of least gradient in metric measure spaces. Rev. Mat. Iberoam. 35(6), 1603-1648 (2019)

16. Koskela, P., Soto, T., Wang, Z.: Traces of weighted function spaces: dyadic norms and Whitney extensions. Sci. China Math. 60(11), 1981-2010 (2017)

17. Koskela, P., Nguyen, K., Wang, Z.: Trace operators on regular trees. Anal. Geom. Metr. Spaces 8(1), 396-409 (2020)

18. Koskela, P., Wang, Z.: Dyadic norm Besov-type spaces as trace spaces on regular trees. Potential Anal. 53(4), 1317-1346 (2020)

19. Lahti, P., Li, X., Wang, Z.: Traces of Newton-Sobolev, hajłasz-sobolev, and BV functions on metric spaces, accepted by Ann. Sc. Norm. Super. Pisa Cl. Sci. (5). arXiv:1911.00533

20. Malý, L.: Trace and extension theorems for Sobolev-type functions in metric spaces. arXiv:1704.06344

21. Malý, L., Shanmugalingam, N., Snipes, M.: Trace and extension theorems for functions of bounded variation. Ann. Sc. Norm. Super. Pisa Cl. Sci. (5) 18(1), 313-341 (2018)

22. Mironescu, P., Russ, E.: Traces of weighted Sobolev spaces. Old and New Nonlinear Anal. 119, 354-381 (2015)

23. Nguyen, K., Classification criteria for regular trees. arXiv:2009.11761

24. Nicase, S., Semin, A.: Density and trace results in generalized fractal networks. ESAIM Math. Model. Numer. Anal. 52(3), 1023-1049 (2018)

25. Peetre, J.: A counterexample connected with Gagliardo's trace theorem, Special issue dedicated to władysław Orlicz on the occasion of his seventy-fifth birthday, Comment. Math. Special Issue 2 , 277-282 (1979)

26. Soardi, P.M.: Potential Theory on Infinite Networks Lecture Notes in Mathematics, vol. 1590. Springer, Berlin (1994)

27. Shanmugalingam, N.: Newtonian spaces: An extension of Sobolev spaces to metric measure spaces. Rev. Mat. Iberoam. 16, 243-279 (2000)

28. Troyanov, M.: Parabolicity of manifolds. Siberian Adv. Math. 9(4), 125-150 (1999)

29. Tyulenev, A.I.: Description of traces of functions in the Sobolev space with a Muckenhoupt weight. Proc. Steklov Inst. Math. 284(1), 280-295 (2014)

30. Tyulenev, A.I.: Traces of weighted Sobolev spaces with Muckenhoupt weight, The case $p=1$. Nonlinear Anal. 128, 248-272 (2015)

31. Villani, A.: Another note on the inclusion $L^{p}(\mu) \subset L^{q}(\mu)$. Amer. Math. Monthly 92(7), 485-487 (1985)

32. Yamasaki, M.: Parabolic and hyperbolic infinite networks. Hiroshima Math. J. 7(1), 135-146 (1977)

33. Wang, Z.: Characterization of trace spaces on regular trees via dyadic norms. J. Math. Anal. Appl. 494(2), 124646, $21(2021)$

Publisher's Note Springer Nature remains neutral with regard to jurisdictional claims in published maps and institutional affiliations. 